\title{
A plastidial retrograde-signal potentiates biosynthesis of systemic stress response activators
}

3 Liping Zeng ${ }^{1 \#}$, Jin-Zheng Wang ${ }^{1 \#}$, Xiang $\mathrm{He}^{2}$, Haiyan $\mathrm{Ke}^{1}$, Mark Lemos ${ }^{1}$, William M. Gray ${ }^{3}$,

4 Katayoon Dehesh ${ }^{1 *}$

5

$6{ }^{1}$ Institute for Integrative Genome Biology and Department of Botany and Plant Sciences,

7 University of California, Riverside, CA 92521

$8{ }^{2}$ Current address: Laboratory of Allergy and Inflammation, Chengdu third people's hospital

9 branch of National Clinical Research Center for Respiratory Disease, Chengdu 610031, China

$10{ }^{3}$ Department of Plant and Microbial Biology, University of Minnesota, St. Paul, MN 55108

11

12 *Author for correspondence:

13 Katayoon Dehesh

14 Email:kdehesh@ucr.edu

$15{ }^{\#}$ The authors contributed equally to this work.

16

17

\begin{tabular}{|c|c|c|c|}
\hline $\begin{array}{l}\text { Total word count (including } \\
\text { Introduction, Materials and } \\
\text { Methods, Results and } \\
\text { Discussion) }\end{array}$ & 6322 & No. of figures: & 9 (Figs $3,5,6,9$ in color) \\
\hline Summary & 160 & No. of tables: & 0 \\
\hline Introduction & 1256 & $\begin{array}{l}\text { No. of Supporting } \\
\text { Information files: }\end{array}$ & $\begin{array}{l}16 \text { (Fig. S1-S9; Table S1-S3; } \\
\text { Supplemental data sets 1-4) }\end{array}$ \\
\hline Materials and Methods: & 1164 & & \\
\hline Results: & 2918 & & \\
\hline Discussion: & 984 & & \\
\hline Acknowledgements: & 94 & & \\
\hline
\end{tabular}




\section{Summary}

- Plants employ an array of intricate and hierarchical signaling cascades to perceive and transduce informational cues to synchronize and tailor adaptive responses. Systemic stress response (SSR) is a recognized complex signaling and response network quintessential to plant's local and distal responses to environmental triggers, however, the identity of the initiating signals has remained fragmented.

- Here, we show that both biotic (aphids and viral pathogens) and abiotic (high-light and wounding) stresses induce accumulation of the plastidial-retrograde-signaling metabolite, methylerythritol cyclodiphosphate (MEcPP), leading to reduction of the phytohormone, auxin, and the subsequent decreased expression of the phosphatase, PP2C.D1.

- This enables phosphorylation of mitogen-activated protein kinases (MAPK3/6), and the consequential induction of the downstream events ultimately resulting in biosynthesis of the two SSR priming metabolites, pipecolic- and N-hydroxy-pipecolic acid.

- This work identifies plastids as the initiation site, and the plastidial retrograde-signal, MEcPP as the initiator of a multi-component signaling cascade potentiating the biosynthesis of SSR activators, in response to biotic and abiotic triggers.

\section{Key Words:}

MAPK3/6, MEcPP, N-hydroxy-pipecolic acid, pipecolic acid, plastidial retrograde-signal, PP2C.D1

1

2

3

44

5

6

7

8

9

0




\section{Introduction}

Dynamic organization of strata of intertwined signaling circuitries is fundamental to the integrity of cellular homeostasis in response to informational cues. Stress responses are induced via intricate and highly organized tiers of signaling cascades where the deactivation/activation of one component potentiates interaction and function of another. Uncovering the nature, the organization, and the operational mode of these tiered communication networks is one of the prime challenges of biology.

A well-recognized key mechanism in the transduction of intracellular signals in eukaryotic organisms is transmission of information via posttranslational protein modifications, most notably reversible protein phosphorylation carried out by protein kinases and protein phosphatases. Protein phosphatases are classified into three groups, among them type 2C protein phosphatases (PP2Cs), a structurally unique class of $\mathrm{Mg}^{2+}-\mathrm{Mn}^{2+}$-dependent enzymes (Olsen et al., 2006; Moorhead et al., 2007; Fuchs et al., 2013). The Arabidopsis genome encodes eighty PP2Cs, nine of which belong to the D-subclade (Fuchs et al., 2013). Initial computational analyses of PP2C.D proteins identified a putative bipartite nuclear localization signal in all nine family members together with a potential transmembrane spanning region in PP2C.D1, D3, D4, D6, D7, and D9 (Schweighofer et al., 2004). Subsequent studies using protein-GFP reporters noted exclusive presence of PP2C.D2, D5, and D6 on the plasma membrane, detected D1, D3, and D4 in the nuclear and cytosolic compartments, and D8 in mitochondria (Ren et al., 2018). In addition to the distinct localization patterns, phosphatases are implicated in different functions including regulation of apical hook development (Sentandreu et al., 2011; Spartz et al., 2014), auxin-induced cell expansion (Spartz et al., 2014; Ren et al., 2018; Wang, J et al., 2020), leaf senescence (Xiao et al., 2015), immune responses (Couto et al., 2016), and altered intracellular responses to exogenous and endogenous stimulus via their nuclear/cytosolic interactions with mitogen-activated protein kinases (MAPKs) (Schweighofer et al., 2007; Umbrasaite et al., 2010;

77 Galletti et al., 2011; Fuchs et al., 2013). In Arabidopsis MAPKs are divided into four (A-D) groups (Ichimura et al., 2002). Group A includes MAPK3, MAPK6, and their orthologs, activated by phosphorylation in response to biotic and abiotic stimuli and by developmental cues (Kiegerl et al., 2000; Zhang \& Klessig, 81 2001; Ichimura et al., 2002; Seo et al., 2007). Specifically, a range of stressors such as 
the nucleus where they reconfigure transcriptional landscape by phosphorylating transcription factors (Kovtun et al., 2000; Miles et al., 2005; Pitzschke \& Hirt, 2009; Taj et al., 2010). Intriguingly, the activation of MAPK3/6 result in the induction of selected stress-response genes, and block the action of auxin, thus providing a link between oxidative stress and auxin signal transduction (Kovtun et al., 2000).

Auxin [indole-3-acetic acid (IAA)] is an indispensable morpho-regulatory hormone involved in integration of developmental and environmental signals into a complex regulatory network permitting optimal architectural modifications in response to the prevailing conditions (Gil et al., 2001; Cheong et al., 2002; Navarro et al., 2006; Spaepen et al., 2007; Kazan \& Manners, 2009). As such, auxin homeostasis is key to refinement of plant responses to an array of environmental signals such as ROS (Laskowski et al., 2002; Zhong et al., 2010; Tognetti et al., 2012; Yu et al., 2013). Interestingly, the proposed connection between auxin and plastid-to-nucleus (retrograde) signaling implied primary function of plastidial retrograde signal in auxin-based signaling cascade (Glasser et al., 2014). Indeed, recently the methylerythritol phosphate (MEP)-pathway intermediate, methylerythritol cyclodiphosphate (MEcPP), was identified as the stress-specific retrograde signaling metabolite, modulating growth by reducing the abundance of auxin and its transporter PIN1 via dual transcriptional and posttranslational regulatory inputs in response to abiotic stresses (Jiang et al., 2018). The connection provided solid evidence for the primary role of plastids in establishing a balance between plant growth and stress responses in accordance to the prevailing conditions (Jiang et al., 2018; Jiang et al., 2019; Jiang et al., 2020). In addition, auxin is also a known key constituent of the phytohormone-based signaling network mediating the regulation of defense responses, as evident by the suppression of the majority of the auxin responsive genes after induction of systemic acquired resistance (SAR) (Wang et al., 2007; Verma et al., 2016).

Whole plant immunity, coined SAR, is the process of priming defense responses in leaves distal to the local infection (Hunt et al., 1996; Ryals et al., 1996). This process is central to a broadspectrum immunity protecting plants from immediate and future biotic attacks (Pieterse et al., 2009; Leon-Reyes et al., 2010; Spoel \& Dong, 2012). However, pathogens are not unique in their ability to elicit systemic signals, since abiotic stresses also induce the rapidly transmitted systemic signal(s) from local to distal leaves, a response known as systemic acquired acclimation (SAA), key to acclimatory responses and improved tolerance (Karpinski et al., 1999; Czarnocka 
114

115

116

117

118

119

120

121

122

123

124

125

126

127

128

129

130

131

132

133

134

135

136

137

138

139

140

141

142

143

et al., 2020; Zandalinas et al., 2020). It is of note that both SAR and SAA, the two seemingly independent systemic responses, are triggered by common stress signals such as ROS (Baxter et al., 2014). This is to be expected since the establishment of SAR is not independent of abiotic cues such as light (Zeier et al., 2004), suggestive of overlapping regulatory components between the two networks.

Long distance communication and signal amplification of SAR is triggered by a number of mobile metabolites including salicylic acid (SA), methyl salicylate (MeSA), a lipid-transfer protein designated defective in induced resistance, azelaic acid, glycerol-3-phosphate, pipecolic acid (Pip) and its derivative N-hydroxy-pipecolic acid (NHP) (Jung et al., 2009; Chanda et al., 2011; Navarova et al., 2012; Chen et al., 2018). Specifically, Pip and NHP are noted signaling molecules that control both SA-dependent and SA-independent SAR activation, and are indispensable for the establishment of nearly all the respective transcriptional responses (Bernsdorff et al., 2016). Pip is synthesized by the agd2-like defense response protein1 (ALD1) (Navarova et al., 2012; Ding et al., 2016; Hartmann et al., 2017). Subsequent N-hydroxylation of Pip by flavin-dependent monooxygenase 1 (FMO1) results in formation of NHP (Chen et al., 2018; Hartmann, M. et al., 2018). Ultimately, elevation of Pip and NHP levels enable the establishment of SAR associated priming responses (Navarova et al., 2012; Zeier, 2013; Ding et al., 2016; Chen et al., 2018). Interestingly however, a recent study shows that FMO1 also plays an important role in triggering of an SAA response, supporting the notion that SAA and SAR not only respond to the same triggers, but also share part or all steps of the same signaling pathway(s) (Baxter et al., 2014; Czarnocka et al., 2020).

Despite numerous reports on the establishment of systemic signaling, the identity and the complexity of the initiating signals potentiating this key defense/adaptive mechanism remains elusive. Here, the exploitation of genetically manipulated lines that either inducibly or constitutively (cehl mutant) accumulate MEcPP, together with biotically and abiotically stressed plants accumulating MEcPP, aided us to uncover the organizational sequence and the mode of MEcPP-mediated action in potentiating a multi-component cascade responsible for the production metabolites that trigger a general systemic signaling responses (SSR). The sequence of these events commences by MEcPP-mediated reduction of auxin abundance and the consequential decreased expression of auxin response factors $(A R F \mathrm{~s})$, the transcriptional 
activators of PP2C.D1. The resulting reduction of PP2C.D1 transcripts enables phosphorylation of MAPK3 and 6, and the consequential induction of events leading to biosynthesis of Pip and NHP, the two key activators of SSR triggered by abiotic (wounding and high light) and biotic (aphid and a vital pathogen) stresses.

Collectively our finding establishes MEcPP as an initiator of the SSR to defend plants against a myriad of environmental challenges.

\section{Materials and Methods}

\section{Plant material and growth condition}

Arabdopsis thaliana seedlings were grown in 16-h light/8-h dark cycles at $\sim 22{ }^{\circ} \mathrm{C}$. Two-weekold seedling were treated with DEX, MEcPP $(100 \mu \mathrm{M})$, IAA $(10 \mu \mathrm{m}, 1 \mathrm{hr})$, Luciferase $(1 \mathrm{mM})$, high light $\left(800 \mu \mathrm{mol} \mathrm{m} \mathrm{sec}^{-1}\right)$ or wounded by forceps as described previously (Benn et al., 2016; Jiang et al., 2018). The pp2c mutant (SALK_099356) was obtained from TAIR. Seedlings were grown under $12 \mathrm{~h}$ light photoperiod at $22-24{ }^{\circ} \mathrm{C}$ for aphids infestation.

\section{Phylogenetic analyses}

Protein sequences of PP2C Clade-D family (Xue et al., 2008) were downloaded from Phytozome. The software MEGA (Kumar et al., 2018) and PhyML (Guindon et al., 2010) was performed to construct the phylogeny.

\section{Luciferase-Activity quantification}

Luciferase activity signals were detected by a CCD camera (Wang et al., 2014). Quantification and statistical analyses of RSRE:LUC activity were performed (Benn et al., 2014).

\section{Metabolites extraction and analyses}

MEcPP, IAA and Salicylic acid were analyzed as previously described (Jiang et al., 2019).

Pip measurements were performed using a Dionex Ultimate 3000 binary RSLC system coupled to Thermo Q-Exactive Focus mass spectrometer with a heated electro spray ionization source. Plant samples were separated using an Accucore-150-Amide-HILIC column (150 X $2.1 \mathrm{~mm}$; particle size $2.6 \mu \mathrm{M}$; Thermo Scientific 16726-152130) with a guard column containing the same column matrix (Thermo Scientific 852-00; 16726-012105). Gradient elution was carried out with 
171

172

173

174

175

176

177

178

179

180

181

182

183

184

185

186

187

188

189

190

191

192

193

194

195

196

197

198

199

200

acetonitrile (A) and $10 \mathrm{mM}$ ammonium acetate $\mathrm{pH} 7.0$ (B). The separation was conducted using the gradient profile $(t(\min ), \% \mathrm{~A}, \% \mathrm{~B}):(-2,90,10),(0,90,10),(12,30,70),(15,30,70),(16,90$, 10), $(22,90,10)$. The flow rate was kept at $280 \mu \mathrm{L} / \mathrm{min}$ and the injected volume was $2 \mu \mathrm{L}$. The column was kept at $35{ }^{\circ} \mathrm{C}$. Mass spectra were acquired in positive mode under the following parameters: spray voltage, $4.50 \mathrm{KV}$; sheath gas flow rate 50 , auxiliary gas flow rate 14 , sweep gas flow rate 2, capillary temperature of $275^{\circ} \mathrm{C}$, S-lens RF level 100 and auxiliary gas heater temperature $275^{\circ} \mathrm{C}$. The initial $0.5 \mathrm{~min}$ of each run was sent to waste to avoid salt contamination of the MS. Compounds of interest were identified by accurate mass measurements (MS1), retention time and mass transitions monitoring. Pip was identified by using standard (Sigma, P45850). For relative quantitation, peak area for each compound (MS1; Thermo Trace Finder Software) was normalized to the initial fresh weight mass.

$N$-OH-Pip was measured using the same system as Pip measurements. Plant samples were separated by an Acquity UPLC HSS T3 column (1.8- $\mu \mathrm{m}, 150 \mathrm{X} 2.1 \mathrm{~mm})$ (Waters, part \# 186003539). The mobile phases were $\mathrm{A}(\mathrm{ACN}, 0.1 \% \mathrm{FA})$ and $\mathrm{B}$ (water, 0.1\% FA), and the gradient was implemented at a flowrate of $0.2 \mathrm{~mL} / \mathrm{min}$ (percentages indicate percent B): 0-1 min (99\%), 1-8 $\min (99-50 \%), 8-10 \min (50 \%), 10-10.5 \min (50-99 \%)$, and 10.5-13 $\min (99 \%)$. The column was kept at $35^{\circ} \mathrm{C}$. The MS was run in positive ion mode with the following parameters: spray voltage, $4.50 \mathrm{KV}$; sheath gas flow rate 45, auxiliary gas flow rate 20, sweep gas flow rate 2, capillary temperature of $250{ }^{\circ} \mathrm{C}$, S-lens RF level 50 and auxiliary gas heater temperature $250{ }^{\circ} \mathrm{C}$. The initial 1 min of each run was sent to waste to avoid salt contamination of the MS. NHP was accurately identified by using the standard obtained from Professor Elizabeth Sattely's lab at Stanford university, and by accurate mass measurements (MS1), retention time and mass transitions. For relative quantitation, peak area for each compound (MS1; Thermo Trace Finder Software) was normalized to the initial fresh weight mass.

\section{RNA-seq Analysis}

Two-week-old $A$. thaliana seedlings were collected. RNA-seq libraries construction followed the BrAD-seq method (Townsley et al., 2015). Each genotype has six biological replicates. 75bases of single-end reads were sequenced. Tophat2 (Kim et al., 2013) was used to map reads to the genome of $A$. thaliana. DESeq2 (Love et al., 2014) was used to count and normalize mapped reads. Genes with 2 -fold altered expression levels and $p$-value $\leq 0.05$ were identified as 
201

202

203

204

205

206

207

208

209

210

211

212

213

214

215

216

217

218

219

220

221

222

223

224

225

226

227

228

differentially expressed genes. The GO term enrichment analyses were obtained by agriGOv2, and the heatmap was generated by the pheatmap (Kolde \& Kolde, 2015) in $R$ program (Team, 2013) (Table S2). RNA-seq data of SAR and Pip response genes were downloaded from published (Hartmann et al., 2017). List of genes with RSRE-containing promoters were obtained from published (Benn et al., 2016).

\section{Quantification of gene expression}

RT-qPCR was performed as described previously (Walley et al., 2007). The control genes was AT4G26410. Table S3 listed primer sequences.

\section{Agro-infiltration-based transient assays in Nicotiana benthamiana}

$N$. benthamiana transient assay was used to identify the protein-protein interaction between $P P 2 C$ and $M A P K 3$, and MAPK6. pENTR/D-TOPO (Invitrogen) and Gateway systems were used for constructing vectors. Vectors containing $\mathrm{C} / \mathrm{N}$-terminal luciferase fused with $P P 2 C, M A P K 3$ and MAPK6 were introduced into Agrobacterium GV3101 and subsequently used for infiltration of $N$. benthamiana leaves, followed by luciferase activity signal detection using CCD camera (Wang et al., 2014).

\section{Co-Immunoprecipitation}

Two-week-old seedlings were ground in liquid nitrogen and suspended in $2 \mathrm{x}$ extraction buffer (50 mM Tris- $\mathrm{HCl}$ at $\mathrm{pH} 7.5,150 \mathrm{mM} \mathrm{NaCl}, 10 \%$ glycerol, $0.1 \%$ NP-40, protease inhibitor cocktail and phosphatase inhibitor) at $4{ }^{\circ} \mathrm{C}$ for $30 \mathrm{~min}$. The protein suspensions were then centrifuged at 4,000g for $10 \mathrm{~min}$ and filtered out the precipitation using the $100 \mu \mathrm{m}$ Nylon Mesh. The supernatant was incubated with GFP-Trap magnetic beads (for IP) and bmab-20 (negative control) (Chromotek), respectively, for $2 \mathrm{~h}$ at $4{ }^{\circ} \mathrm{C}$. The beads were washed five times with the $2 \mathrm{x}$ extraction buffer. The immuno-precipitants were eluted with 2x SDS lysis buffer (50 mM Tris$\mathrm{HCl}$ at $\mathrm{pH}$ 6.8, 2\% SDS, 10\% glycerol, 0.1\% bromophenol blue, 1\% 2-mercaptoethanol), boiled $\left(100{ }^{\circ} \mathrm{C}, 10 \mathrm{~min}\right)$, separated on SDS-PAGE gel and subsequently transferred onto nitrocellulose membrane for probing with the corresponding antibodies.

\section{Protein extraction and immuno-blot analyses}

Two-week-old seedlings were ground in liquid nitrogen and suspended in 2x SDS lysis buffer 
229

230

231

232

233

234

235

236

237

238

239

240

241

242

243

244

245

246

247

248

249

250

251

252

253

254

255

and boiled (100 $\left.{ }^{\circ} \mathrm{C}, 10 \mathrm{~min}\right)$. Proteins were then separated on SDS-PAGE gel and transferred onto the nitrocellulose membrane. The monoclonal anti-PP2C (1:5000) was previously reported (Spartz et al., 2014). The phosphorylated MAPK3 and MAPK6 proteins were detected using polyclonal anti-phospho-p44/42 MAPK (Erk1/2, 1:1000; Cell Signaling Technology), and the detection of the total MAPK3 and 6 protein levels were by using polyclonal anti-MAPK3 (1:1000, Sigma) and anti-MAPK6 (1:1000, Sigma). The goat-anti-Rabbit (1:3000) HRPconjugated secondary antibody was used.

\section{Aphid infestation}

The potato aphids (Macrosiphum euphorbiae) isolate WU11 (Teixeira et al., 2018) were maintained on their adapted hosts for over 2.5 years in a growth chamber at $20^{\circ} \mathrm{C}$ with $16 \mathrm{~h} \mathrm{light}$ photoperiod. To infest new Arabidopsis seedlings, the colony was released to the growth chamber with the experimental plants to allow the infestation (Teixeira et al., 2018).

\section{Viral infection}

Four-week-old seedlings were infected with cucumber mosaic virus (CMV-m2b) for 2 weeks.

\section{Accession Numbers and RNA-seq data}

PP2C.D1 (AT5G02760), HDS (AT5G60600), MAPK3 (AT3G45640), MAPK6 (AT2G43790), SARD1 (AT1G73805), CBP60g (AT5G26920), ALD1 (AT2G13810), FMO1 (AT1G19250), ARF7 (AT5G20730), ARF19 (AT1G19220), ARF2 (AT5G62000), ARF3 (AT2G33860), ARF10 (AT2G28350), ARF11 (AT2G46530), ARF18 (AT3G61830).

All RNA-seq data were submitted to NCBI SRA database (PRJNA596287).

\section{Results}

\section{MEcPP-mediated transcriptional suppression of PP2C.D1}

Comparative RNA-seq analyses of the high MEcPP-accumulating mutant, ceh1, versus wild-type plant revealed altered expression profile of the clade D phosphatases (Fig. S1a-b). Subsequent studies specifically identified PP2C.D1, also known as APD7 (Arabidopsis PP2C clade D7) or SSPP (senescence-suppressed protein phosphatase) (Tovar-Mendez et al., 2014; Xiao et al., 2015), as the phosphatase with the most notably reduced transcript levels compared to the other 
clade members in the cehl mutant. For simplicity throughout the paper, we will refer PP2C.D1 as PP2C. Indeed, qRT-PCR analyses confirmed markedly lower PP2C expression levels in cehl compared with the wild-type (Fig. 1a).

Next, we analyzed the PP2C expression levels in salicylic acid deficient eds16 and cehl/eds 16 mutants to assess the potential regulatory input of the high SA present in cehl mutant (Xiao et al., 2012) (Fig. 1a). The results illustrate the SA-independent reduction of $P P 2 C$ transcript levels in the high MEcPP-accumulating cehl mutant backgrounds.

To investigate the MEcPP-mediated reduction of $P P 2 C$ transcript levels, we exploited a dexamethasone (DEX)-inducible MEcPP accumulating line (HDSi), previously shown to accumulate similar MEcPP levels as that found in the cehl mutant, at $72 \mathrm{~h}$ post DEX-induction (Jiang et al., 2018; Jiang et al., 2019; Wang, JZ et al., 2020). The analyses of PP2C expression levels in mock- and DEX-treated plants (72h post induction) display an inverse correlation between DEX-inducible accumulation of MEcPP and expression levels of PP2C (Fig. 1b).

To provide a direct evidence for MEcPP-mediated suppression of $P P 2 C$ expression, we examined the relative transcript levels of the gene in mock- and exogenously MEcPP-treated wild-type plants (Fig. 1c). Indeed, the reduced transcript levels of $P P 2 C$ an hour post MEcPP application confirm specificity of MEcPP in mediating this suppression.

To assess whether MEcPP-mediated suppression of $P P 2 C$ is via transcriptional and/or posttranscriptional modifications, we employed plants expressing $P P 2 C$ under the control of the constitutive promoter, 35S:PP2C-GFP (for simplicity herein designated $O E-P P 2 C$ ) and the introgressed line in the ceh1 mutant background (ceh1/OE-PP2C) (Fig. 1d). The similarly high $P P 2 C$ transcripts in $O E-P P 2 C$ and ceh $1 / O E-P P 2 C$ as compared to the notably reduced levels in the cehl mutant background is a clear demonstration of the MEcPP-mediated transcriptional suppression of PP2C. Moreover, immunoblot analyses using PP2C specific antibody established the concordance between the protein and transcript levels, as evidenced by similarly high PP2C protein abundance in $O E-P P 2 C$ and ceh $1 / O E-P P 2 C$ compared to undetectable protein levels in the ceh1 mutant (Figs. 1d and S2).

To examine a potential link between $P P 2 C$ transcript levels and production of SA and/or MEcPP, we examined the abundance of these two metabolites in various genotypes (WT, cehl, ceh1/OE- 
285

286

287

288

289

290

291

292

293

294

295

296

297

298

299

300

301

302

303

304

305

306

307

308

309

310

311

312

313

314

$P P 2 C, O E-P P 2 C$, and the $p p 2 c$ mutant) (Fig. 1e). The data explicitly confirm the PP2Cindependent accumulation of MEcPP and SA.

Collectively, the finding establishes a SA-independent but MEcPP-dependent transcriptional suppression of $P P 2 C$, and excludes any $\mathrm{PP} 2 \mathrm{C}$ regulatory input in accumulation of MEcPP and SA.

\section{MEcPP-mediated transcriptional regulation of $P P 2 C$ is auxin-dependent}

To dissect the regulatory components of $P P 2 C$ transcriptional machinery, we examined and identified four auxin response cis-elements (AuxRE) (Ulmasov et al., 1995) in the PP2C promoter sequences (Fig. 2a). The presence of these auxin-dependent regulatory elements together with reduced abundance of auxin and its transporter PIN1 (Jiang et al., 2018) via the MEcPP-mediated transcriptional and posttranslational regulatory input, led us to examine the $P P 2 C$ transcript levels in mock- and auxin-treated cehl and WT plants (Fig. 2b). The elevated $P P 2 C$ transcript levels in WT and the cehl mutant an hour post IAA-treatment compared with untreated plants is an indicative of IAA-dependent transcriptional regulation of $P P 2 C$ expression, corroborating the previous finding (Nemhauser et al., 2006; Ren et al., 2018). It is of note that lower levels of $P P 2 C$ expression in IAA-treated cehl relative to the corresponding WT is likely in part due to the impairment of auxin distribution in the cehl mutant caused by reduced abundance of auxin transporter, PIN1 (Jiang et al., 2018). Alternatively and or additionally, the reduced expression of $P P 2 C$ in IAA-treated cehl relative to that of the WT plant could be attributed to decreased expression levels of auxin response factors (ARFs), a family of transcription factors responsible for the induction of AuxREs (Ulmasov et al., 1999; Guilfoyle \& Hagen, 2001). To test this hypothesis, we examined expression levels of several family members of $A R F$ s (Figs. 2c and S3). Among the tested members, only $A R F 7$ and 19 displayed reduced transcript levels in cehl backgrounds (cehl and ceh1/eds16) compared to the corresponding controls (WT and eds 16). This prompted us to examine the PP2C expression levels in mock- and auxin-treated single and double arf7 and 19 mutants (Fig. 2d). The partial induction of $P P 2 C$ expression in auxin-treated single mutants as opposed to no induction in the double mutant line compared with the WT plant, establishes the key function of two auxin response factors, ARF7 and 19, in induction of $P P 2 C$.

The data collectively delineate the molecular strata of MEcPP-mediated suppression of $P P 2 C$ 
expression, commenced by reduced abundance of auxin, and the consequential decreased expression of $A R F 7$ and 19 , the $P P 2 C$ transcriptional activators.

\section{$P P 2 C$ suppresses the MEcPP-inducible RSRE-containing stress-response genes}

To examine the consequences of altered $P P 2 C$ transcript levels in high MEcPP containing plants, we analyzed the global expression profiles of $c e h 1 / O E-P P 2 C$ and $H D S i / O E-P P 2 C$ versus those corresponding to cehl and HDSi backgrounds (Supplemental data sets 1-3). The analyses revealed a notable presence (47-to-68\%) of robustly suppressed genes in ceh1/OE-PP2C and $H D S i / O E-P P 2 C$ backgrounds, respectively, that contain a general stress response (GSR) ciselement, coined Rapid Stress Response Element (RSRE) (Walley et al., 2007; Benn et al., 2014; Benn et al., 2016), in their promoters (Fig. 3a, Table S1). To examine the validity of these analyses in planta, we employed 4xRSRE:Luciferase line, used for functional readout of stressinduced rapid transcriptional responses (Walley et al., 2007; Benn et al., 2014; Bjornson et al., 2014), and introgressed it into the cehl and ceh1/OE-PP2C backgrounds. Subsequent luciferase activity assays using homozygous introgressed lines showed markedly reduced luciferase activity in ceh1/OE-PP2C/RSRE:LUC line (herein designated as ceh1/OE-PP2C) compared with the previously established high and constitutive expression of the RSRE in ceh1/RSRE:LUC line (cehl) (Benn et al., 2016) (Fig. 3b). Moreover, additional bioinformatics analyses revealed a 27\% increase in the number of induced stress-response genes containing RSRE in $p p 2 c$ mutant compared to $O E-P P 2 C$ line (Fig. 3c). Combined in vivo and in silico analyses support the involvement of PP2C in transcriptional regulation of RSRE-containing stress response genes.

Specifically, the Gene Ontology enrichment analyses of differentially expressed genes in $p p 2 c$ mutant versus $O E-P P 2 C$ lines revealed transcriptional profile that is partitioned into two distinct clusters of stress-response and growth-related genes (Fig. S4 and Table S2). The inverse expression profiles of the two clusters support the notion of PP2C function in induction of growth-related genes, and suppression of stress-response genes (Fig. S4). Additional analyses established significant enrichment of induced plant-pathogen interaction pathway genes in $p p 2 c$ mutant compared to the wild-type (Fig. S5a). This data support the recent report on reduction of PP2C transcript levels in response to flg22 and nlp20 treatment (Bjornson et al., 2021) (Fig. S5b).

Collectively, our findings uncover the PP2C-mediated transcriptional reconfiguration of RSRE 
containing genes, and further allude to the growth optimizing function of this phosphatase in concordance with its suggested role in promoting apical hook development in etiolated seedlings (Sentandreu et al., 2011; Spartz et al., 2014). Our experimental and bioinformatics data extend supports to the notion of PP2C function in both biotic and abiotic stress responses, and as a governing module balancing growth versus adaptive responses.

\section{PP2C suppresses transcription of Pip and NHP biosynthesis genes and their metabolites}

Extended bioinformatics analyses unraveled a significant reduction in the number of SAR- (43to-52\%) and Pip-induced (44-to-40\%) genes in ceh1/OE-PP2C and HDSi/OE-PP2C versus their corresponding backgrounds, respectively (Fig. S6a-b and Table S1). This together with the known functions of Pip and NHP in triggering SAR (Navarova et al., 2012; Hartmann, Michael et al., 2018), prompted us to test the potential involvement of MEcPP, SA, and PP2C in modulating the expression of genes involved in Pip and NHP biosynthesis. Specifically, we analyzed the relative expression levels of SARD1, CBP60g, ALD1 and FMO1 in WT, cehl, eds16, ceh1/eds16, HDSi, HDSileds16, PP2C overexpressing WT (OE-PP2C), ceh1 (ceh1/OE$P P 2 C$ ) and $p p 2 c$ lines (Fig. 4a-b). Similar expression profiles of the aforementioned genes in the cehl mutant and the DEX-induced HDSi line relative to the corresponding controls (WT and mock-treated $H D S i$ ) illustrate their MEcPP-dependent induction in constitutive and in inducible lines (Fig. 4b). However, while MEcPP induces expression of all the genes (SARD1, CBP60g, $A L D 1$ and $F M O 1$ ), SA differentially alters their expression profile, as evidenced by the reduced SARDI but induced FMO1 expression levels in SA-deficient cehl/eds16 line compared to ceh1. Moreover, the SA-mediated reduction of CBP6Og in the inducible line is hindered by constitutive production of MEcPP, whereas the $A L D 1$ transcript levels remain SA-independent (Fig. 4b).

Additional studies show that the overexpression of PP2C (ceh1/OE-PP2C) diminishes the MEcPP-mediated transcriptional induction of Pip and NHP biosynthetic genes, albeit at different degrees (Fig. 4b). It is noteworthy that higher transcript levels of these genes in cehl and ceh1/OE-PP2C relative to the WT, OE-PP2C, or pp2C may be due to the elevated MEcPP in the ceh1 mutant. Lastly, similarly low expression levels of the genes in $p p 2 c$ and WT lines could be attributed to the standard as opposed to stressed growth condition.

Next, we profiled Pip and NHP metabolite levels in aforementioned genotypes employed in the transcriptional profiling (Fig. 4c). In concordance with the altered transcriptional profiles, 
375 accumulation of Pip and NHP metabolites is positively correlated to the presence of constitutively high or inducible MEcPP levels in cehl and HDSi lines (Fig. 4c). Whereas, the two metabolites are differentially accumulated in response to SA as evidenced by the reduced Pip content and enhanced NHP levels in cehl/eds16 relative to the cehlmutant. In addition, decreased Pip and NHP levels in the ceh1/OE-PP2C relative to the ceh1 mutant clearly support the PP2C-mediated reduction of both metabolites in spite of the high MEcPP levels.

Collectively, the finding establishes PP2C-mediated transcriptional suppression of Pip and NHP biosynthesis genes and by extension reduction of their corresponding metabolites, critical for eliciting systemic responses.

\section{PP2C interacts with MAPK3 and 6}

To examine the subcellular site of PP2C action in high MEcPP containing cehl mutant, we imaged the PP2C-GFP tagged $O E-P P 2 C$ and ceh1/OE-PP2C lines, and confirmed plasma membrane, cytosolic and nuclear localization of the protein as previously reported for the WT background (Spartz et al., 2014; Tovar-Mendez et al., 2014; Ren et al., 2018) (Fig. 5a).

To identify PP2C protein targets, we initially employed two independent methods. One method was based on immunoprecipitation-mass spectrometry (IP-MS) using a GFP specific antibody for IP of the PP2C interacting proteins in ceh1/OE-PP2C and $O E-P P 2 C$ lines, and the control wild-type plant. As a second method, we employed a yeast-two-hybrid library-screening assay. The subsequent MS profiling of the samples derived from each of these two methods (Supplemental dataset 4), led to identification of several PP2C interacting proteins, most notably among them MAPK3 and 6.

Because of the indispensable function of these MAPKs in triggering Pip accumulation and by extension SAR induction (Wang et al., 2018), we verified their interactions with PP2C by additional methods. One method was based on the agro-infiltration-based transient assays in Nicotiana benthamiana. For this approach we used fusion constructs of MAPK3/6 and PP2C in various configurations (MAPK3 fused to carboxyl-terminal fragment of LUC, and PP2C fused to amino-terminal fragment of LUC, MAPK6 fused to amino-terminal fragment and PP2C fused to carboxyl-terminal fragment of LUC) (Figs. 5b and S7a-b). The luciferase reconstitution-based activity is clearly evident in the leaves co-infiltrated with $P P 2 C$ and $M A P K 3$ and 6 fusion 
constructs, but is absent in the leaves co-infiltrated with the respective controls. In a second independent approach, we examined the in vivo interaction of PP2C with MAPK3 and MAPK6 by targeted co-immunoprecipitation (CO-IP) using a GFP specific antibody for IP of PP2C-GFP in ceh1/OE-PP2C, OE-PP2C and $p P P 2 C: P P 2 C-G F P$ lines, followed by immunoblot analyses using GFP as well as the MAPK3 and MAPK6 specific antibodies (Fig. 5c). The clear and specific presence of an MAPK3 and MAPK6 reacting bands in the IP fractions of ceh1/OE$P P 2 C$ and $O E-P P 2 C$ lines, but not in the control agarose beads, verified the in vivo interaction of PP2C with MAPK3 and MAPK6 proteins (Fig. 5c).

Next, we explored the ramification of the interaction between MAPKs and PP2C protein by comparing the levels of phosphorylated MAPK3 and 6 in various genotypes (WT, ceh1, ceh1/OE-PP2C, pp2c, and OE-PP2C) using $\alpha$-pMAPK6 and $\alpha$-pMAPK3 antibodies deemed to specifically detect the respective phosphorylated proteins. The immunoblots clearly show similarly abundant phosphorylated kinases in WT and $p p 2 c$, however the levels are slightly but detectably reduced in $O E-P P 2 C$ line grown under standard conditions (Fig. 5d). Furthermore, these differences are not attributed to changes in the total MAPK3/6 protein abundance in WT, pp $2 c$ and $O E-P P 2 C$ line, as they display similar levels on the immunoblots probed with the respective antibodies (Fig. 5d). The most notable reduction in the abundance of phosphorylated MAPK3 and 6 is in ceh1/OE-PP2C compared to ceh1 (Fig. 5d). It is of note that the MAPK3 protein levels are similar between cehl and ceh1/OE-PP2C, albeit more abundant than that of the other genotypes. This difference in abundance could contribute to higher levels of phosphorylated MAPK3 in the ceh1 mutant relative to other genotypes, namely WT, $p p 2 c$ and $O E-P P 2 C$. However, the higher level of phosphorylated MAPK3 in ceh1 compared to ceh1/OE$P P 2 C$ is in spite of the similar abundance of total proteins in these genotypes. Additionally, altered levels of phosphorylated MAPK6 in various genotypes, most notably with heightened abundance in the ceh1 mutant, is seemingly independent of slight variation of MAPK6 total protein levels amongst these genotypes (Fig. 5d).

The above results collectively identify MAPK3 and 6 kinases as PP2C interacting proteins. Moreover, the inverse correlation between PP2C protein abundance and the phosphorylated levels of MAPK3/6 support the notion of PP2C function as the phosphatase.

\section{Abiotic and biotic stresses enhance MEcPP and induce Pip and NHP levels}


434 We have previously established that the two most prevalent environmental insults, wounding and

435

436

437

438

439

440

441

442

443

444

445

446

447

448

449

450

451

452

453

454

455

456

457

458

459

460

461

462

463

high-light induce MEcPP accumulation in plants (Xiao et al., 2012). This data together with MEcPP-mediated increased levels of Pip and NHP metabolites prompted us to examine the impact of mechanical damage and high-light on accumulation of these two SAR triggering metabolites. Thus, we examined the sequential steps of the events starting from MEcPP accumulation to the production of Pip and NHP in high-light treated and wounded seedlings.

We initially examined high-light treated plants and established their increased MEcPP and decreased auxin content using transgenic R2D2 reporter lines expressing auxin-degradable (DII) fluorescent protein as a proxy for IAA levels (Liao et al., 2015) (Figs 6a-b). Reduced IAA content prompted us to examine the expression levels of $P P 2 C$ and the auxin-responsive transcription factors, $A R F 7$ and 19 in control and stressed seedlings (Fig. 6c). Reduced expression levels of these genes in response to high-light treatment are reminiscent of our earlier observation in the cehl mutant (Fig. 2c). Reduced $A R F 7$ and 19 transcript levels in stressed seedlings prompted us to examine the ramification of these reductions on the expression of genes within Pip and NHP biosynthetic pathway. As such, we compared the transcript levels of CBP60, ALDI and FMO1 in the WT and arf7/19 mutant lines (Fig. S8). The clear enhancement of the transcript levels of all genes in the arf7/19 mutant line alludes to the suppressive function of ARF9 and 17 on the expression of Pip and NHP biosynthetic-pathway genes. The result provides a rationale for the stress-mediated suppression of ARF7 and 19, hence enabling increased production of Pip and NHP.

Next, we examined the relative abundance of phosphorylated MAPK3 and 6 in the high-light treated seedlings compared to the control (Fig. 6d). The data establishes similar abundance of their total proteins but enhanced levels of phosphorylated MAPK3/6 under high light condition, (Fig. 6d). Next we compared the abundance of Pip and NHP metabolites in the stressed versus the control seedlings and confirmed high-light-induced accumulation of the metabolites (Fig. 6e). The finding supports the notion of Pip and NHP triggering SAA in response to high-light exposure.

Subsequently, we extended these studies to include unwounded and wounded (90 min post wounding) $p p 2 c$ mutant and wild-type plants. The finding clearly establishes wound-induced MEcPP-accumulation independently of PP2C (Fig. 7a). Furthermore, similarly to high-light- 
464 treated plants, auxin levels as well as the expression levels of $A R F 7,19$ and $P P 2 C$ are reduced in 465 wounded relative to unwounded plants (Fig. 7b-c). Moreover, wounding induces 466 phosphorylation of MAPK3 and 6 in the WT and $p p 2 c$ mutant (Figs. 7d and S9). Accordingly, 467 there is increased accumulation of Pip and NHP in both backgrounds albeit at higher levels in 468 pp2c compared to the WT (Fig. 7e).

469 Next we examined the multi-component signaling cascade potentiating the biosynthesis of SSR 470 in plants challenged with two biotic stresses, aphids (Macrosiphum euphorbiae) and a viral 471 pathogen [Cucumber mosaic virus $(C M V-m 2 b)$ ] (Fig. 8a-d). The data clearly show increased 472 MEcPP levels upon aphid infestation and CMV infection, followed by decreased expression of $473 A R F 7 / 19$ and $P P 2 C$, and the ensued heightened MAPK3 and 6 phosphorylation albeit without 474 altered abundance of the total proteins, and finally induction of Pip and NHP metabolites.

475 Collectively, our findings establish a link between the two prevalent naturally occurring abiotic stresses and biotic insults to the induction of MEcPP levels followed by the reduction in auxin abundance and the consequential decline in PP2C expression, and ultimately phosphorylation of MAPK3/6 and accumulation of Pip and NHP metabolites. As such, the data supports functional expansion of Pip and NHP in eliciting SSR by abiotic or biotic triggers.

\section{Discussion}

Plants have evolved complex tiers of molecular and biochemical networks to detect, transmit and amplify adaptive signals for dynamic restoration of cellular homeostasis and function at the local and the distal site of insults. This broad spectrum response known as systemic stress response (SSR) appears to be conserved in plants across the plant kingdom, and as such the focus of intense studies (Shah \& Zeier, 2013). However, the identity of signals that initiate this response has remained fragmentary. Here, we provide a complete module of the nature and the sequence of events that trigger SSR cascade (Fig. 9). Specifically, we illustrate that accumulation of the stress-specific plastidial retrograde signaling metabolite, MEcPP, achieved either by genetic manipulation or via challenging plants with the two prevalent abiotic challenges (mechanical damage and high-light treatment) and two biotic insults (aphids and CMV), result in reduced auxin concentration and the consequential decreased expression of $A R F 7$ and 19, the 492 transcriptional activators of $P P 2 C$. Auxin-induction of $P P 2 C$ corroborates the earlier finding 493 (Nemhauser et al., 2006) and supports the notion of PP2C function as a suppressor of stress- 
response genes and an inducer of the growth-related genes. Although the notion is contradicted by the report of auxin-induced SMALL AUXIN UP-RNA binding to, and inhibiting SAUR19. This results in inactivation of PP2C and consequential phosphorylation and hence activity of $\mathrm{H}+-$ ATPase required for cell expansion in the apical hook of etiolated seedlings, where PP2C is abundantly present (Spartz et al., 2014; Ren et al., 2018). Indeed, induction of PP2C expression by auxin and suppression of its enzyme activity by the auxin-induced SAUR19 represent two conflicting auxin-based regulatory responses. These opposing responses are prime examples of multi-layered auxin-based fine-tuning of PP2C both at the expression and at the enzyme activity levels. This delicate balance shifts the function of PP2C enzyme to either a positive or a negative regulator of growth, depending upon the tissue, and tailored to nature of the environmental challenges.

Combined parallel and independent approaches of IP-MS, yeast-two-hybrid library screening, split-luciferase assay and CO-IP followed by immuno-blot analyses verified physical interactions between MAPK3/6 and PP2C protein. The biological ramification of this interaction is best captured by enhanced levels of phosphorylated MAPK3 and 6 in the constitutively (ceh1 mutant) and stress-inducible MEcPP accumulating plants, and conversely by their reduced phosphorylated forms in ceh1/OE-PP2C lines, raising the likelihood of PP2C function as the responsible phosphatase. This notion is supported by the reduction of MAPK3/6 phosphorylation in $O E-P P 2 C$ line compared to WT. It is of note that the lack of enhanced MAPK3/6 phosphorylation in $p p 2 c$ mutant line under standard condition, is likely due to the absence of a stress-activated kinase within the MAPK kinase kinases cascade, otherwise present in MEcPP accumulating cehl mutant, and in biotically and abiotically challenged plants. This scenario is supported by $\mathrm{H}_{2} \mathrm{O}_{2}$ activation of ANP1, an Arabidopsis MAPKKK that initiates a phosphorylation cascade including MAPK3/6 followed by induction of specific stress-responsive genes, and suppression of auxin-inducible genes (Kovtun et al., 2000). Indeed, the functional input of MAPKs in mis-localizing polar auxin transport proteins (PINs) expands the regulatory roles of activated MAPKs in hampering auxin distribution and signal transduction (Jia et al., 2016; Dory et al., 2018). This process may therefore constitute the underlying mechanism of reduced abundance of PIN1 in the MEcPP accumulating cehl mutant, where MAPK phosphorylation of PIN1 result in mis-localization and degradation of this auxin transporter (Jiang et al., 2018). If so, this places the reduction of PP2C transcript and the consequential 
525 activation of MAPK3/6 at the interface between MEcPP and auxin signaling, and uncovers the sequence of events between the two regulatory capacities required for tailoring plant growth and

527 developmental responses to environmental cues.

528 The converse correlation between PP2C abundance and the prevalence of SAR inducible genes in MEcPP-accumulating plants, confirmed by qRT-PCR analyses of targeted genes involved in Pip and NHP biosynthesis genes, is in agreement with the increase of the respective metabolite levels. Furthermore, analyses of constitutive and inducible MEcPP accumulating plant that are either deficient in, or contain high SA, show differential SA-mediated transcriptional responses. That is, except for the SARD1 whose induction is partly dependent on SA, the expression levels of the other three genes are either SA-independent $(A L D 1)$ or suppressed by SA (CBP60g and FMO1). In contrast, MEcPP induces the expression of all these genes albeit at different degrees, placing SARD1 as the least and FMO1 as the most MEcPP-responsive genes. Moreover, analyses of Pip and NHP metabolite levels show that while SA induces Pip production, it suppresses NHP levels, whereas MEcPP-mediates induction of both metabolites although at different levels. This establishes MEcPP as the inducer of SSR, and further suggests that despite the critical role of SA as a mobile signal for SAR (Neuenschwander et al., 1995; Park et al., 2007), SA is not essential

541 for production of Pip and NHP.

542 Additionally, increased levels of Pip and NHP in plants challenged with wounding and high-light 543 expands the role of these SAR triggering metabolites to the establishment of a resistance state 544 not only when confronted with biotic but also when challenged with abiotic insults as evidenced by the reported FMOI induction in response to $\mathrm{H}_{2} \mathrm{O}_{2}$ accumulation (Chen \& Umeda, 2015). In summary, here we reveal the nature and the organization of a multicomponent retrograde

547 signaling cascade that induces the biosynthesis of Pip and NHP in response to both biotic and abiotic insults. This occurs through alterations of positive and negative regulators that enable a timely modification of expression profiles and the consequential reconfiguration of the metabolic network for optimal implementation of this adaptive response as a general strategy to fend against a complex myriad of insults. We specifically identify plastids as the initiation site and the

552 plastidial retrograde signaling metabolite, MEcPP, as the initiating signal potentiating the 553 concerted arrays of signaling network responsible for production of Pip and NHP, the triggers of systemic stress responses in face of a myriad of environmental challenges. 
We would like to thank Professor Elizabeth Sattely at Stanford University for providing us with the chemically synthesized NHP standard. We would like to thank our colleagues at UCR, Prof. Isgouhi Kaloshian and Dr. Jacob Macwilliams for aphid-infested plants, and Dr. Bailong Zhang for viral-infected plants. This work was supported by NSF CREATE-IGERT training program (NSF DGE-0653984) and NSF-GRFP 1148897 to M.L, by Dr. John W. Leibacher and Mrs. Kathy Cookson endowed chair funds to $\mathrm{KD}$ and by National Institutes of Health (NIH) GM067203 to W.M.G., and by NIH, R01GM107311-8 grant to KD.

\section{Authors' contributions}

L. Z., J.Z.W. and KD designed the study, L.Z., J.Z.W., X.H., H. K. and M. L. performed the experiments, W. M. G. provided antibody and K.D. wrote the manuscript.

\section{REFERENCES}

Baxter A, Mittler R, Suzuki N. 2014. ROS as key players in plant stress signalling. Journal of Experimental Botany 65(5): 1229-1240.

Benn G, Bjornson M, Ke H, De Souza A, Balmond EI, Shaw JT, Dehesh K. 2016. Plastidial metabolite MEcPP induces a transcriptionally centered stress-response hub via the transcription factor CAMTA3. Proceedings of the National Academy of Sciences of the United States of America 113(31): 8855-8860.

Benn G, Wang CQ, Hicks DR, Stein J, Guthrie C, Dehesh K. 2014. A key general stress response motif is regulated non-uniformly by CAMTA transcription factors. The Plant journal : for cell and molecular biology 80(1): 82-92.

Bernsdorff F, Döring A-C, Gruner K, Schuck S, Bräutigam A, Zeier J. 2016. Pipecolic acid orchestrates plant systemic acquired resistance and defense priming via salicylic aciddependent and-independent pathways. The Plant Cell 28(1): 102-129.

Bjornson M, Benn G, Song X, Comai L, Franz AK, Dandekar AM, Drakakaki G, Dehesh K. 2014. Distinct roles for mitogen-activated protein kinase signaling and CALMODULIN-BINDING TRANSCRIPTIONAL ACTIVATOR3 in regulating the peak time and amplitude of the plant general stress response. Plant Physiol 166(2): 988996.

Bjornson M, Pimprikar P, Nurnberger T, Zipfel C. 2021. The transcriptional landscape of Arabidopsis thaliana pattern-triggered immunity. Nat Plants.

Chanda B, Xia Y, Mandal MK, Yu K, Sekine KT, Gao QM, Selote D, Hu Y, Stromberg A, Navarre D, et al. 2011. Glycerol-3-phosphate is a critical mobile inducer of systemic immunity in plants. Nat Genet 43(5): 421-427.

Chen P, Umeda M. 2015. DNA double-strand breaks induce the expression of flavin-containing monooxygenase and reduce root meristem size in Arabidopsis thaliana. Genes Cells 20(8): 636-646.

Chen YC, Holmes EC, Rajniak J, Kim JG, Tang S, Fischer CR, Mudgett MB, Sattely ES. 2018. N-hydroxy-pipecolic acid is a mobile metabolite that induces systemic disease 
resistance in Arabidopsis. Proceedings of the National Academy of Sciences of the United States of America 115(21): E4920-E4929.

Cheong YH, Chang HS, Gupta R, Wang X, Zhu T, Luan S. 2002. Transcriptional profiling reveals novel interactions between wounding, pathogen, abiotic stress, and hormonal responses in Arabidopsis. Plant Physiol 129(2): 661-677.

Couto D, Niebergall R, Liang X, Bucherl CA, Sklenar J, Macho AP, Ntoukakis V, Derbyshire P, Altenbach D, Maclean D, et al. 2016. The Arabidopsis Protein Phosphatase PP2C38 Negatively Regulates the Central Immune Kinase BIK1. PLoS Pathog 12(8): e1005811.

Czarnocka W, Fichman Y, Bernacki M, Różańska E, Sańko-Sawczenko I, Mittler R, Karpiński S. 2020. FMO1 Is Involved in Excess Light Stress-Induced Signal Transduction and Cell Death Signaling. Cells 9(10): 2163.

Ding P, Rekhter D, Ding Y, Feussner K, Busta L, Haroth S, Xu S, Li X, Jetter R, Feussner I, et al. 2016. Characterization of a Pipecolic Acid Biosynthesis Pathway Required for Systemic Acquired Resistance. The Plant cell 28(10): 2603-2615.

Dory M, Hatzimasoura E, Kallai BM, Nagy SK, Jager K, Darula Z, Nadai TV, Meszaros T, Lopez-Juez E, Barnabas B, et al. 2018. Coevolving MAPK and PID phosphosites indicate an ancient environmental control of PIN auxin transporters in land plants. FEBS letters 592(1): 89-102.

Fuchs S, Grill E, Meskiene I, Schweighofer A. 2013. Type 2 C protein phosphatases in plants. FEBS J 280(2): 681-693.

Galletti R, Ferrari S, De Lorenzo G. 2011. Arabidopsis MPK3 and MPK6 play different roles in basal and oligogalacturonide- or flagellin-induced resistance against Botrytis cinerea. Plant physiology 157(2): 804-814.

Gil P, Dewey E, Friml J, Zhao Y, Snowden KC, Putterill J, Palme K, Estelle M, Chory J. 2001. BIG: a calossin-like protein required for polar auxin transport in Arabidopsis. Genes Dev 15(15): 1985-1997.

Glasser C, Haberer G, Finkemeier I, Pfannschmidt T, Kleine T, Leister D, Dietz KJ, Hausler RE, Grimm B, Mayer KFX. 2014. Meta-Analysis of Retrograde Signaling in Arabidopsis thaliana Reveals a Core Module of Genes Embedded in Complex Cellular Signaling Networks. Mol Plant 7(7): 1167-1190.

Guilfoyle TJ, Hagen G. 2001. Auxin response factors. Journal of Plant Growth Regulation 20(3): 281-291.

Guindon S, Dufayard J-F, Lefort V, Anisimova M, Hordijk W, Gascuel O. 2010. New algorithms and methods to estimate maximum-likelihood phylogenies: assessing the performance of PhyML 3.0. Systematic biology 59(3): 307-321.

Hartmann M, Kim D, Bernsdorff F, Ajami-Rashidi Z, Scholten N, Schreiber S, Zeier T, Schuck S, Reichel-Deland V, Zeier J. 2017. Biochemical Principles and Functional Aspects of Pipecolic Acid Biosynthesis in Plant Immunity. Plant physiology 174(1): 124153.

Hartmann M, Zeier T, Bernsdorff F, Reichel-Deland V, Kim D, Hohmann M, Scholten N, Schuck S, Bräutigam A, Hölzel T. 2018. Flavin monooxygenase-generated Nhydroxypipecolic acid is a critical element of plant systemic immunity. Cell 173(2): 456469. e416.

Hartmann M, Zeier T, Bernsdorff F, Reichel-Deland V, Kim D, Hohmann M, Scholten N, Schuck S, Brautigam A, Holzel T, et al. 2018. Flavin Monooxygenase-Generated N- 
Hydroxypipecolic Acid Is a Critical Element of Plant Systemic Immunity. Cell 173(2): 456-469 e416.

Hunt MD, Neuenschwander UH, Delaney TP, Weymann KB, Friedrich LB, Lawton KA, Steiner HY, Ryals JA. 1996. Recent advances in systemic acquired resistance research-a review. Gene 179(1): 89-95.

Ichimura K, Shinozaki K, Tena G, Sheen J, Henry Y, Champion A, Kreis M, Zhang SQ, Hirt H, Wilson C, et al. 2002. Mitogen-activated protein kinase cascades in plants: a new nomenclature. Trends in plant science 7(7): 301-308.

Jia W, Li B, Li S, Liang Y, Wu X, Ma M, Wang J, Gao J, Cai Y, Zhang Y, et al. 2016. Mitogen-Activated Protein Kinase Cascade MKK7-MPK6 Plays Important Roles in Plant Development and Regulates Shoot Branching by Phosphorylating PIN1 in Arabidopsis. PLoS Biol 14(9): e1002550.

Jiang J, Rodriguez-Furlan C, Wang JZ, de Souza A, Ke H, Pasternak T, Lasok H, Ditengou FA, Palme K, Dehesh K. 2018. Interplay of the two ancient metabolites auxin and MEcPP regulates adaptive growth. Nature communications 9(1): 2262.

Jiang J, Xiao Y, Chen H, Hu W, Zeng L, Ke H, Ditengou FA, Devisetty U, Palme K, Maloof J, et al. 2020. Retrograde Induction of phyB Orchestrates Ethylene-Auxin Hierarchy to Regulate Growth. Plant physiology 183(3): 1268-1280.

Jiang J, Zeng L, Ke H, De La Cruz B, Dehesh K. 2019. Orthogonal regulation of phytochrome $\mathrm{B}$ abundance by stress-specific plastidial retrograde signaling metabolite. Nat Commun 10(1): 2904.

Jung HW, Tschaplinski TJ, Wang L, Glazebrook J, Greenberg JT. 2009. Priming in systemic plant immunity. Science 324(5923): 89-91.

Karpinski S, Reynolds H, Karpinska B, Wingsle G, Creissen G, Mullineaux P. 1999. Systemic signaling and acclimation in response to excess excitation energy in Arabidopsis. Science 284(5414): 654-657.

Kazan K, Manners JM. 2009. Linking development to defense: auxin in plant-pathogen interactions. Trends Plant Sci 14(7): 373-382.

Kiegerl S, Cardinale F, Siligan C, Gross A, Baudouin E, Liwosz A, Eklof S, Till S, Bogre L, Hirt H, et al. 2000. SIMKK, a mitogen-activated protein kinase (MAPK) kinase, is a specific activator of the salt stress-induced MAPK, SIMK. The Plant cell 12(11): 22472258.

Kim D, Pertea G, Trapnell C, Pimentel H, Kelley R, Salzberg SL. 2013. TopHat2: accurate alignment of transcriptomes in the presence of insertions, deletions and gene fusions. Genome biology 14(4): R36.

Kolde R, Kolde MR. 2015. Package 'pheatmap'. R Package 1(7): 790.

Kovtun Y, Chiu WL, Tena G, Sheen J. 2000. Functional analysis of oxidative stress-activated mitogen-activated protein kinase cascade in plants. Proceedings of the National Academy of Sciences of the United States of America 97(6): 2940-2945.

Kumar S, Stecher G, Li M, Knyaz C, Tamura K. 2018. MEGA X: molecular evolutionary genetics analysis across computing platforms. Molecular biology and evolution 35(6): 1547-1549.

Laskowski MJ, Dreher KA, Gehring MA, Abel S, Gensler AL, Sussex IM. 2002. FQR1, a novel primary auxin-response gene, encodes a flavin mononucleotide-binding quinone reductase. Plant Physiol 128(2): 578-590.

Leon-Reyes A, Van der Does D, Koornneef A, Van Wees SC, Pieterse CM. 2010. 
Networking by small-molecule hormones in plant immunity. Phytopathology 100(6): S160-S160.

Liao CY, Smet W, Brunoud G, Yoshida S, Vernoux T, Weijers D. 2015. Reporters for sensitive and quantitative measurement of auxin response. Nat Methods 12(3): 207-210, $202 \mathrm{p}$ following 210.

Love MI, Huber W, Anders S. 2014. Moderated estimation of fold change and dispersion for RNA-seq data with DESeq2. Genome biology 15(12): 550.

Miles GP, Samuel MA, Zhang Y, Ellis BE. 2005. RNA interference-based (RNAi) suppression of AtMPK6, an Arabidopsis mitogen-activated protein kinase, results in hypersensitivity to ozone and misregulation of AtMPK3. Environ Pollut 138(2): 230-237.

Moorhead GB, Trinkle-Mulcahy L, Ulke-Lemee A. 2007. Emerging roles of nuclear protein phosphatases. Nat Rev Mol Cell Biol 8(3): 234-244.

Navarova H, Bernsdorff F, Doring AC, Zeier J. 2012. Pipecolic Acid, an Endogenous Mediator of Defense Amplification and Priming, Is a Critical Regulator of Inducible Plant Immunity. The Plant cell 24(12): 5123-5141.

Navarro L, Dunoyer P, Jay F, Arnold B, Dharmasiri N, Estelle M, Voinnet O, Jones JD. 2006. A plant miRNA contributes to antibacterial resistance by repressing auxin signaling. Science 312(5772): 436-439.

Nemhauser JL, Hong F, Chory J. 2006. Different plant hormones regulate similar processes through largely nonoverlapping transcriptional responses. Cell 126(3): 467-475.

Neuenschwander U, Vernooij B, Friedrich L, Uknes S, Kessmann H, Ryals J. 1995. Is Hydrogen-Peroxide a 2nd-Messenger of Salicylic-Acid in Systemic Acquired-Resistance. Plant Journal 8(2): 227-233.

Olsen JV, Blagoev B, Gnad F, Macek B, Kumar C, Mortensen P, Mann M. 2006. Global, in vivo, and site-specific phosphorylation dynamics in signaling networks. Cell 127(3): 635648.

Park SW, Kaimoyo E, Kumar D, Mosher S, Klessig DF. 2007. Methyl salicylate is a critical mobile signal for plant systemic acquired resistance. Science 318(5847): 113-116.

Pieterse CMJ, Leon-Reyes A, Van der Ent S, Van Wees SCM. 2009. Networking by smallmolecule hormones in plant immunity. Nat Chem Biol 5(5): 308-316.

Pitzschke A, Hirt H. 2009. Disentangling the complexity of mitogen-activated protein kinases and reactive oxygen species signaling. Plant physiology 149(2): 606-615.

Ren H, Park MY, Spartz AK, Wong JH, Gray WM. 2018. A subset of plasma membranelocalized PP2C.D phosphatases negatively regulate SAUR-mediated cell expansion in Arabidopsis. PLoS genetics 14(6): e1007455.

Ryals JA, Neuenschwander UH, Willits MG, Molina A, Steiner HY, Hunt MD. 1996. Systemic Acquired Resistance. The Plant cell 8(10): 1809-1819.

Schweighofer A, Hirt H, Meskiene I. 2004. Plant PP2C phosphatases: emerging functions in stress signaling. Trends in plant science 9(5): 236-243.

Schweighofer A, Kazanaviciute V, Scheikl E, Teige M, Doczi R, Hirt H, Schwanninger M, Kant M, Schuurink R, Mauch F. 2007. The PP2C-type phosphatase AP2C1, which negatively regulates MPK4 and MPK6, modulates innate immunity, jasmonic acid, and ethylene levels in Arabidopsis. The Plant cell 19(7): 2213-2224.

Sentandreu M, Martin G, Gonzalez-Schain N, Leivar P, Soy J, Tepperman JM, Quail PH, Monte E. 2011. Functional profiling identifies genes involved in organ-specific branches of the PIF3 regulatory network in Arabidopsis. The Plant cell 23(11): 3974-3991. 
Seo S, Katou S, Seto H, Gomi K, Ohashi Y. 2007. The mitogen-activated protein kinases WIPK and SIPK regulate the levels of jasmonic and salicylic acids in wounded tobacco plants. Plant Journal 49(5): 899-909.

Shah J, Zeier J. 2013. Long-distance communication and signal amplification in systemic acquired resistance. Front Plant Sci 4.

Spaepen S, Vanderleyden J, Remans R. 2007. Indole-3-acetic acid in microbial and microorganism-plant signaling. FEMS Microbiol Rev 31(4): 425-448.

Spartz AK, Ren H, Park MY, Grandt KN, Lee SH, Murphy AS, Sussman MR, Overvoorde PJ, Gray WM. 2014. SAUR inhibition of PP2C-D phosphatases activates plasma membrane $\mathrm{H}+-\mathrm{ATPases}$ to promote cell expansion in Arabidopsis. The Plant Cell 26(5): 2129-2142.

Spoel SH, Dong XN. 2012. How do plants achieve immunity? Defence without specialized immune cells. Nature Reviews Immunology 12(2): 89-100.

Taj G, Agarwal P, Grant M, Kumar A. 2010. MAPK machinery in plants: recognition and response to different stresses through multiple signal transduction pathways. Plant Signal Behav 5(11): 1370-1378.

Team RC 2013. R: A language and environment for statistical computing: Vienna, Austria.

Teixeira MA, Sela N, Atamian HS, Bao E, Chaudhary R, MacWilliams J, He J, Mantelin S, Girke T, Kaloshian I. 2018. Sequence analysis of the potato aphid Macrosiphum euphorbiae transcriptome identified two new viruses. PloS one 13(3): e0193239.

Tognetti VB, Muhlenbock P, Van Breusegem F. 2012. Stress homeostasis - the redox and auxin perspective. Plant Cell Environ 35(2): 321-333.

Tovar-Mendez A, Miernyk JA, Hoyos E, Randall DD. 2014. A functional genomic analysis of Arabidopsis thaliana PP2C clade D. Protoplasma 251(1): 265-271.

Townsley BT, Covington MF, Ichihashi Y, Zumstein K, Sinha NR. 2015. BrAD-seq: Breath Adapter Directional sequencing: a streamlined, ultra-simple and fast library preparation protocol for strand specific mRNA library construction. Frontiers in plant science 6: 366.

Ulmasov T, Hagen G, Guilfoyle TJ. 1999. Activation and repression of transcription by auxinresponse factors. Proceedings of the National Academy of Sciences of the United States of America 96(10): 5844-5849.

Ulmasov T, Liu ZB, Hagen G, Guilfoyle TJ. 1995. Composite structure of auxin response elements. The Plant cell 7(10): 1611-1623.

Umbrasaite J, Schweighofer A, Kazanaviciute V, Magyar Z, Ayatollahi Z, Unterwurzacher V, Choopayak C, Boniecka J, Murray JA, Bogre L, et al. 2010. MAPK phosphatase AP2C3 induces ectopic proliferation of epidermal cells leading to stomata development in Arabidopsis. PloS one 5(12): e15357.

Verma V, Ravindran P, Kumar PP. 2016. Plant hormone-mediated regulation of stress responses. BMC Plant Biol 16.

Walley JW, Coughlan S, Hudson ME, Covington MF, Kaspi R, Banu G, Harmer SL, Dehesh K. 2007. Mechanical stress induces biotic and abiotic stress responses via a novel cis-element. PLoS genetics 3(10): 1800-1812.

Wang C-Q, Guthrie C, Sarmast MK, Dehesh K. 2014. BBX19 interacts with CONSTANS to repress FLOWERING LOCUS T transcription, defining a flowering time checkpoint in Arabidopsis. The Plant Cell 26(9): 3589-3602.

Wang D, Pajerowska-Mukhtar K, Culler AH, Dong X. 2007. Salicylic acid inhibits pathogen growth in plants through repression of the auxin signaling pathway. Current biology : $C B$ 
818

819

820

821

17(20): 1784-1790.

Wang J, Sun N, Zhang F, Yu R, Chen H, Deng XW, Wei N. 2020. SAUR17 and SAUR50 Differentially Regulate PP2C-D1 during Apical Hook Development and Cotyledon Opening in Arabidopsis. The Plant cell 32(12): 3792-3811.

Wang JZ, Lei Y, Xiao Y, He X, Liang J, Jiang J, Dong S, Ke H, Leon P, Zerbe P, et al. 2020. Uncovering the functional residues of Arabidopsis isoprenoid biosynthesis enzyme HDS. Proceedings of the National Academy of Sciences of the United States of America 117(1): 355-361.

Wang Y, Schuck S, Wu J, Yang P, Doring AC, Zeier J, Tsuda K. 2018. A MPK3/6WRKY33-ALD1-Pipecolic Acid Regulatory Loop Contributes to Systemic Acquired Resistance. The Plant cell 30(10): 2480-2494.

Xiao D, Cui Y, Xu F, Xu X, Gao G, Wang Y, Guo Z, Wang D, Wang NN. 2015. SENESCENCE-SUPPRESSED PROTEIN PHOSPHATASE Directly Interacts with the Cytoplasmic Domain of SENESCENCE-ASSOCIATED RECEPTOR-LIKE KINASE and Negatively Regulates Leaf Senescence in Arabidopsis. Plant physiology 169(2): 1275-1291.

Xiao Y, Savchenko T, Baidoo EE, Chehab WE, Hayden DM, Tolstikov V, Corwin JA, Kliebenstein DJ, Keasling JD, Dehesh K. 2012. Retrograde signaling by the plastidial metabolite MEcPP regulates expression of nuclear stress-response genes. Cell 149(7): 1525-1535.

Xue T, Wang D, Zhang S, Ehlting J, Ni F, Jakab S, Zheng C, Zhong Y. 2008. Genome-wide and expression analysis of protein phosphatase $2 \mathrm{C}$ in rice and Arabidopsis. $B M C$ genomics 9(1): 550.

Yu X, Pasternak T, Eiblmeier M, Ditengou F, Kochersperger P, Sun J, Wang H, Rennenberg H, Teale W, Paponov I, et al. 2013. Plastid-localized glutathione reductase2-regulated glutathione redox status is essential for Arabidopsis root apical meristem maintenance. Plant Cell 25(11): 4451-4468.

Zandalinas SI, Fichman Y, Devireddy AR, Sengupta S, Azad RK, Mittler R. 2020. Systemic signaling during abiotic stress combination in plants. Proceedings of the National Academy of Sciences of the United States of America 117(24): 13810-13820.

Zeier J. 2013. New insights into the regulation of plant immunity by amino acid metabolic pathways. Plant Cell and Environment 36(12): 2085-2103.

Zeier J, Pink B, Mueller MJ, Berger S. 2004. Light conditions influence specific defence responses in incompatible plant-pathogen interactions: uncoupling systemic resistance from salicylic acid and PR-1 accumulation. Planta 219(4): 673-683.

Zhang S, Klessig DF. 2001. MAPK cascades in plant defense signaling. Trends in plant science 6(11): 520-527.

Zhong R, Thompson J, Ottesen E, Lamppa GK. 2010. A forward genetic screen to explore chloroplast protein import in vivo identifies Moco sulfurase, pivotal for ABA and IAA biosynthesis and purine turnover. Plant J 63(1): 44-59.

\section{Figure legends}




\section{Figure 1. MEcPP-mediates transcriptional suppression of PP2C}

823

824

825

826

827

828

829

830

831

832

833

834

835

836

837

838

839

840

841

842

843

844

845

846

847

848

849

850

(a) Suppression of PP2C expression is MEcPP-dependent and SA-independent. Total RNAs isolated from two-week-old seedlings of wild-type (WT), ceh1/eds16, eds16 were subjected to qRT-PCR analyses. (b) Accumulation of MEcPP is inversely correlated to PP2C transcript levels. Relative expression of PP2C and MEcPP levels in DEX-inducible HDSi line 72 hours post mock- (-) and DEX-treatment (+). Analyses were performed on two-week-old seedlings. (c) Relative expression levels of PP2C in WT plants, 60 min post mock- (-) and MEcPP (100 $\mu \mathrm{M})$ treatment $(+)$ confirms MEcPP-dependent transcriptional suppression of PP2C. (d) Reduced expression level of $P P 2 C$ in cehl is recovered in $P P 2 C$ overexpressing ceh1 (ceh1/OE-PP2C) and wild-type $(O E-P P 2 C)$ lines. Immunoblot analyses using PP2C antibody display undetectable protein in ceh1, but detectably similar levels in PP2C overexpressing lines. The lower nonspecific reacting band and Ponceau S staining show equal loading. (e) Analyses of MEcPP and SA levels in WT, ceh1, ceh1/OE-PP2C, OE-PP2C and PP2C mutant line (pp2c) show PP2Cindependent accumulation of the metabolites. The PP2C mRNA levels was normalized to the levels of At4g26410 (M3E9). All Data are mean \pm SD of three biological and three technical replicates. Two-tailed Student's $t$ tests or ANOVA tests confirm MEcPP-mediated suppression of $P P 2 C$. Asterisks denotes significance. Lower case letters on top of histograms represent statistically significant differences $(P \leq 0.05)$.

\section{Figure 2. Auxin induces PP2C expression}

(a) The schematic presentation of the $P P 2 C$ promoter display the positions of auxin response elements (AuxRE). (b) Expression levels of PP2C an hour post mock- (-) and auxin (10 $\mu \mathrm{M})$ treatment $(+)$ of cehl and WT seedlings display enhanced expression in auxin-treated lines. (c) Reduced expression levels of auxin response factors (ARF7 and 19) in ceh1 and ceh1/eds16 compared to the levels in WT and eds16 lines. (d) PP2C expression levels an hour post mock- (-) and auxin $(10 \mu \mathrm{M})$-treatment $(+)$ of WT, single (arf7, and arf19) and double (arf7/arf19) mutant lines display indispensable function of ARF7 and 19 in auxin-induction of $P P 2 C$ expression. Statistical analyses were performed by two-tailed Student's $t$ tests or ANOVA tests.

\section{Figure 3. PP2C suppresses the MEcPP-inducible SAR and RSRE-containing genes}

(a) Venn diagrams illustrate suppression of a significant number of MEcPP-inducible RSRE- 
851

852

853

854

855

856

857

858

859

860

861

862

863

864

865

866

867

868

869

870

871

872

873

874

875

876

877

878

879

880

motif containing genes in $P P 2 C$ overexpressing (ceh1/OE-PP2C and HDSi/OE-PP2C) lines. (b) Representative images of $P P 2 C$ overexpressing lines in the cehl background (ceh1/OE-PP2C) show the notable reduction of RSRE:LUC activity compared to the cehl mutant. The bar underneath displays the intensity of LUC activity and the histogram show quantification of LUC activity. Data are presented as means \pm SEM. Asterisk notes statistically significant differences $(P \leq 0.05)$ by using two-tailed Student's $t$ test. (c) Venn diagrams illustrate enrichment of RSREmotif containing genes whose expressions are significantly induced in the $p p 2 c$ mutant but not in $O E-P P 2 C$ plants under standard conditions.

Figure 4. PP2C suppresses transcription of Pip and NHP biosynthesis genes and corresponding metabolites

(a) Schematic presentation of pipecolic acid (Pip) and N-hydroxy-pipecolic acid (NHP) biosynthesis pathway. (b) Relative expression levels of SARD1, CBP60g, ALD1 and FMO1 in WT, ceh1, eds16, ceh1/eds16, and in mock (-) and post $72 \mathrm{~h}$ DEX-treated HDSi and HDSi/eds16 lines, and in $P P 2 C$ overexpression in the wild-type (OE-PP2C) and the ceh1 mutant (ceh1/OEPP2C) backgrounds as well as in pp2c mutant, show reversion of SA- and MEcPP-dependent induction of these genes in PP2C overexpressing lines. (c) Measurements of Pip and NHP metabolites in aforementioned genotypes confirm their MEcPP- and SA-dependent alterations, and their reduced abundance in $P P 2 C$ overexpression lines. All Data are mean $\pm \mathrm{SD}$ of three biological and three technical replicates. Lower case letters on top of histograms represent statistically significant differences $(P \leq 0.05)$ by using ANOVA test.

Figure 5. MAPK3/6 phosphorylation levels and their physical interaction with PP2C

(a) Confocal images of plasma membrane, cytosolic and nuclear localization of $\mathrm{PP} 2 \mathrm{C}$ in the wild-type $(O E-P P 2 C)$ and ceh1 (ceh1/OE-PP2C) backgrounds overexpressing 35S::PP2C-GFP construct. (b) Representatives of split luciferase complementation assays in Nicotiana benthamiana displayed by dark-field images of leaves expressing cLuc-MAPK3 (C-terminal Luc fused with MAPK3) and nLuc-PP2C (N-terminal Luc fragment fused with PP2C) (upper panel) and MAPK6-nLuc (MAPK6 fused with N-terminal fragment of Luc) and PP2C-cLuc (PP2C fused with C-terminal fragment Luc) (lower panel). (c) The in vivo interaction of PP2C with MAPK3 and MAPK6 determined by co-immunoprecipitation assay. Protein samples obtained from ceh1/OE-PP2C, OE-PP2C, and $p P P 2 C: P P 2 C-G F P$ seedlings grown under standard 
conditions were immunoprecipitated using GFP (+) and empty (-) magnetic beads. Immunoblots were analyzed using with $\alpha$-MAPK3 or $\alpha$-MAPK6. Each blot shows protein inputs before (input, right panels) and after (IP, left panels) immunoprecipitation. (d) Immunoblots show that PP2C overexpression in the cehl mutant reverses the MEcPP-mediated high phosphorylation of MAPK3 and MAPK6 ( $\alpha$-pMAPK6 and $\alpha$-pMAPK3, top panels), without notable impact on the protein abundance of MAPK6 (middle panels) or MAPK3 (bottom panels). Ponceau S staining shows protein loading.

\section{Figure 6. High light induces MEcPP accumulation and enhances Pip and NHP abundance}

(a) Induction of MEcPP levels and (b) reduction of IAA abundance 90 min post high light (800 $\mu \mathrm{mol} \mathrm{m} \mathrm{m}^{-2}, 90 \mathrm{~min}$ ) treatment as measured by fluorescence of mDII-ntdTomato/DII-n3xVenus and the corresponding bright-field images of control and high light-treated auxin reporter R2D2 seedlings. (c) Relative expression levels of $A R F 7 / 19$ and PP2C in high-light-treated seedlings. (d) Immunoblot analyses of total and phosphorylated MAPK3/6 in high-light-treated seedlings. Ponceau S show equal protein loading. (e) Enhanced levels of Pip and NHP metabolites in high light-treated seedlings. Two-tailed Student's $t$ tests and ANOVA tests are used for the statistical analyses and the asterisk and different letters denote significance $(P \leq 0.05)$.

\section{Figure 7. Wounding induces MEcPP accumulation and enhances Pip and NHP abundance}

(a) Induction of MEcPP levels and (b) reduction of IAA abundance in 90 min post wounded WT and $p p 2 c$ mutant seedlings compared to control unwounded plants.

(c) Relative expression levels of $A R F 7 / 19$ and $P P 2 C$ in unwounded and wounded WT plants. (d) Immunoblot analyses of total and phosphorylated MAPK3/6 in unwounded and wounded WT and pp2c mutant seedlings. Ponceau S show equal protein loading. (e) Metabolic analyses of Pip and NHP in wounded and WT and $p p 2 c$ mutant seedlings. Two-tailed Student's $t$-tests and ANOVA tests are used for the statistical analyses and the asterisk and different letters denote statistical significance $(P \leq 0.05)$.

Figure 8. Aphid infestation and viral infection induce MEcPP accumulation and enhance Pip and NHP abundance

(a) Induction of MEcPP post 2 weeks of cucumber mosaic virus (CMV-M2b) infection, and 24h aphid infestation in WT seedlings, and (b) suppression of $A R F 7 / 19$ and PP2C expression in 
biotically challenged plants. (c) Immunoblot analyses of total and phosphorylated MAPK3/6 in mock (-) and virus/aphids (+) treated seedlings. Ponceau S show equal protein loading. (d) Metabolic analyses of Pip and NHP in mock (-) and virus/aphids (+) treated seedlings. The asterisk denotes statistical significance $(P \leq 0.05)$ by using two-tailed Student's $t$-tests.

\section{Figure 9. Biotic and abiotic insults trigger the retrograde signaling cascade initiating SSR}

Schematic model depicting biotically and abiotically-induced MEcPP-accumulation mediates reduction of auxin abundance that lessens expression levels of the ARF7/19, the transcriptional activators of $P P 2 C$. This enables phosphorylation of MAPK3/6 required for induction of Pip and $\mathrm{NPH}$ biosynthesis genes and production of their respective metabolites key to activation of general SSR.

\section{Supporting Information}

Figure S1. Reduced expression of PP2C in the ceh1 mutant

(a) Phylogeny of PP2C family members in clade D. (b) RNA-seq-based analyses of relative expression levels of PP2C.D family members show decreased levels of PP2C.D1 and PP2C.D7, and increased levels of PP2C.D8 and PP2C.D9 expression in the ceh1 mutant relative to the wild-type plant.

\section{Figure S2. PP2C immunoblot.}

(a) Detection of PP2C protein using PP2C antibody on an immunoblot of protein extracts from various genotypes. (b) Ponseau S stain shows the equal loading.

\section{Figure S3 Relative expression levels of selected $A R F$ family members}

Total RNAs isolated from two-week-old wild-type (WT), ceh1/eds 16, eds16 seedlings were subjected to q-PCR analyses. Relative expression levels of representative $A R F \mathrm{~s}$ (ARF2, $A R F 3$, $A R F 10, A R F 11$ and $A R F 18$ ) were normalized to the levels of At4g26410 (M3E9). All Data are mean $\pm \mathrm{SD}$ of three biological and three technical replicates. Two-tailed Student's $t$ test confirms MEcPP-independent expression of these $A R F$ members.

Figure S4. GO term analyses implicate PP2C as a growth-promoter and a stresssuppressor 
937 Comparative GO term analyses of induced genes in the $p p 2 c$ mutant and $P P 2 C$ overexpressing 938 line $(O E-P P 2 C)$ implicate PP2C as a stress suppressor and a growth promoter. The red bar shows 939 the $-\log _{10} \mathrm{P}$-values of altered transcript levels.

Figure S5. PP2C is likely involved in biotic stress responses.

(a) KEGG pathway enrichment analyses of induced genes in the $p p 2 c$ mutant compared to Col, implicating PP2C as a biotic stress suppressor. The red bar shows the $-\log _{10} \mathrm{P}$-values of enriched pathways. (b) The expression level of $P P 2 C$ is significantly suppressed by pathogenassociated molecular patterns (PAMPs) treatment (flg22 and nlp20) of Col post 90 and $180 \mathrm{~min}$. Data retrieved from the recently published report (Bjornson et al., 2021). The star on each histogram indicates significant changes of the expression level compared to the representative mock treatment.

Figure S6. PP2C overexpression modifies transcriptional profile

Venn diagrams illustrate significantly reduced number of SAR-induced (a) and Pip-induced genes (b) in PP2C overexpressing ceh1 and inducible HDSi lines.

\section{Figure S7. Split luciferase complementation assays in Nicotiana benthamiana}

952 Representatives of split luciferase complementation assays in Nicotiana benthamiana displayed 953 by bright-field images of leaves expressing cLuc-MAPK3 (C-terminal Luc fused with MAPK3) 954 and nLuc-PP2C (N-terminal Luc fragment fused with PP2C) (upper panel) and MAPK6-nLuc 955 (MAPK6 fused with N-terminal fragment of Luc) and PP2C-cLuc (PP2C fused with C-terminal 956 fragment Luc) (lower panel). Negative controls for each constructs include cLuc-MAPK3 \& 957 nLuc; cLuc \& nLuc-PP2C; MAPK6-nLuc \& cLuc and nLuc \& PP2C-cLuc.

959 Total RNAs isolated from two-week-old wild-type and arf7/19 double mutant seedlings were subjected to q-PCR analyses. Relative expression levels of CBP60g, ALD1 and FMO1 were normalized to the levels of At4g26410 (M3E9). All Data are mean \pm SD of three biological and three technical replicates. The star represents the significantly statistic differences by two-tailed Student's $t$ test.

964 Figure S9. Normalized relative intensity of protein levels. 
965 Phosphorylated MAPK6 (pMAPK6) and MAPK3 (pMAPK3), and un-phosphorylated MAPK6

966 and MAPK3 proteins are normalized to the levels of Ponceau stain of Rubisco.

967 Table S1. Percentage of MEcPP-induced and PP2C-supppressed SAR- and Pip-inducible 968 genes, and the RSRE containing genes

969 Table S2. GO term analyses of the up-regulated genes in $p p 2 c$ mutant and $O E-P P 2 C$. The 970 presented number is $-\log 10$ (P-value) for each of the GO term.

971 Table S3. List of used primers

972 Supplemental data sets 1-3. List of differentially expressed genes

973 Supplemental data set 4. List of identified proteins in IP-MS 
a

d

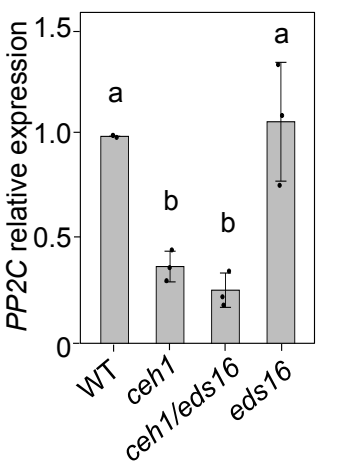

b

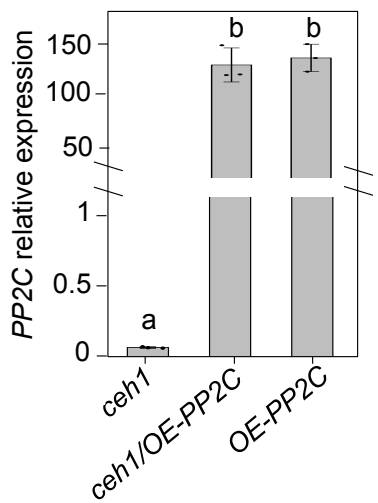

e

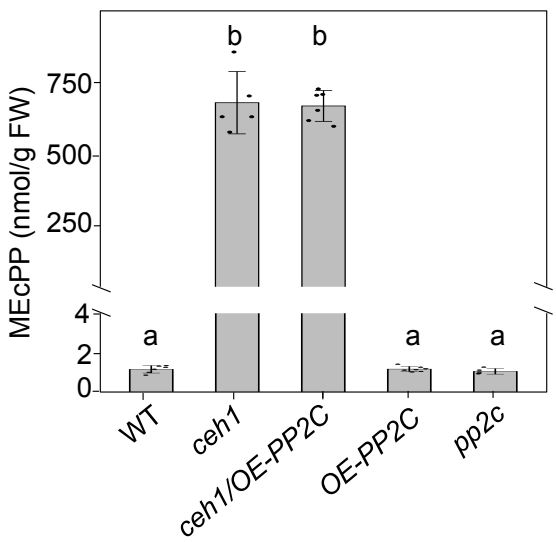

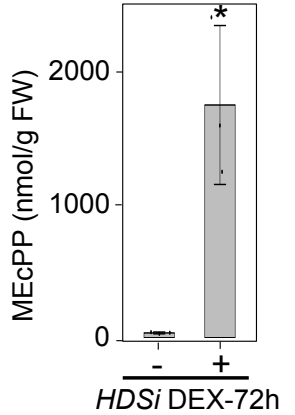

C

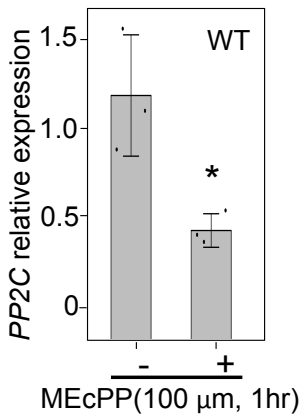

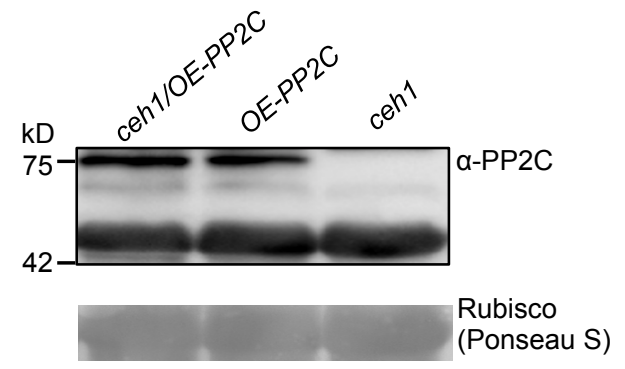

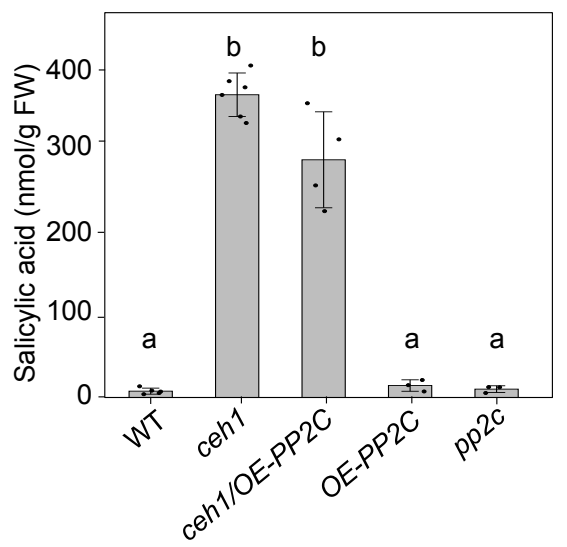

Figure 1. MEcPP-mediates transcriptional suppression of $P P 2 C$

(a) Suppression of PP2C expression is MEcPP-dependent and SA-independent. Total RNAs isolated from two-week-old seedlings of wild type (WT), ceh1/eds16, eds16 were subjected to GRT-PCR analyses. (b) Accumulation of MEcPP is inversely correlated to $P P 2 C$ transcript levels. Relative expression of PP2C and MEcPP levels in DEX-inducible HDSi line 72 hours post mock- (-) and DEX-treatment (+). Analyses were performed on two-week-old seedlings. (c) Relative expression levels of PP2C in WT plants, 60 min post mock- (-) and MEcPP (100 mM)-treatment (+) confirms MEcPP-dependent transcriptional suppression of PP2C. (d) Reduced expression levels of $P P 2 C$ in ceh1 is recovered in $P P 2 C$ overexpressing ceh1 (ceh1/OE-PP2C) and wild type (OE-PP2C) lines. Immunoblot analyses using PP2C antibody display undetectable protein in ceh1, but detectably similar levels in PP2C overexpressing lines. The lower non-specific reacting band and Ponceau S staining show equal loading. (e) Analyses of MEcPP and SA levels in WT, ceh1, ceh1/OE-PP2C, OE-PP2C and PP2C mutant line ( $p p 2 c)$ show PP2C-independent accumulation of the metabolites. The PP2C mRNA levels was normalized to the levels of At4g26410 (M3E9). All Data are mean \pm SD of three biological and three technical replicates. Two-tailed Student's $t$ tests or ANOVA tests confirm MEcPP-mediated suppression of $P P 2 C$. Asterisks denote significance. Lower case letters on top of histograms represent statistically significant differences $(P \leq$ 0.05 ). 
a

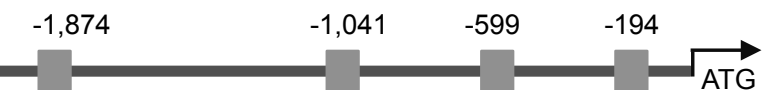

Positions of AuxRE motifs in PP2C promoter

b

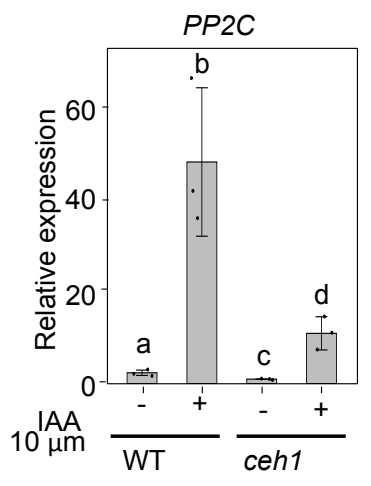

C

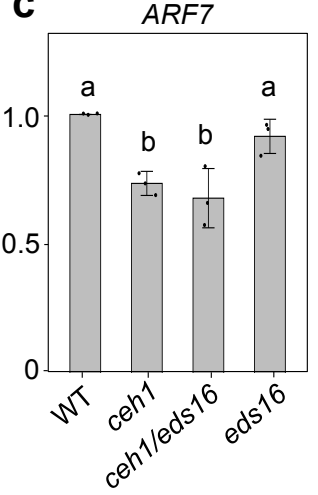

ARF19

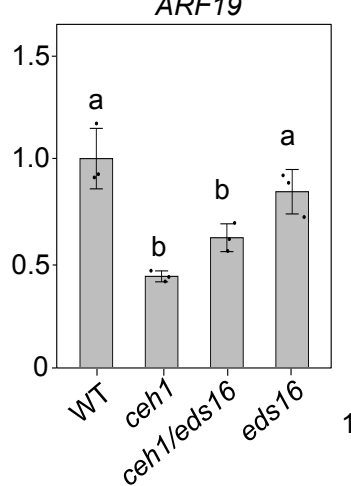

d

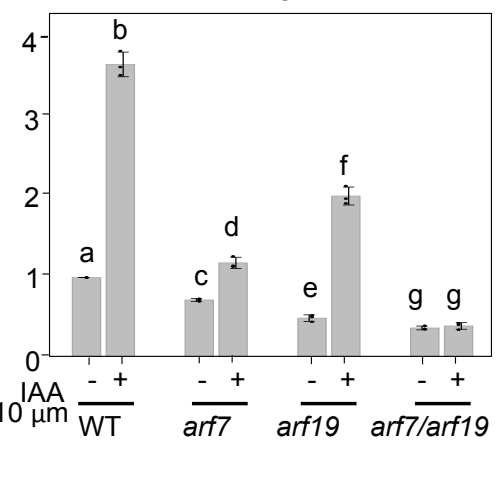

Figure 2. Auxin induces $P P 2 C$ expression

(a) The schematic presentation of the PP2C promoter display the positions of auxin response elements (AuxRE). (b) Expression levels of $P P 2 C$ an hour post mock- (-) and auxin (10 mM) -treatment (+) of ceh1 and WT seedlings display enhanced expression in auxin-treated lines. (c) Reduced expression levels of auxin response factors (ARF7 and 19) in ceh1 and ceh1/eds16 compared to the levels in WT and eds16 lines. (d) PP2C expression levels an hour post mock- (-) and auxin (10 mM)-treatment (+) of WT, single (arf7, and arf19) and double (arf7/arf19) mutant lines display indispensable function of ARF7 and 19 in auxin-induction of PP2C expression. Statistical analyses were performed by two-tailed Student's $t$ tests or ANOVA tests. 
a
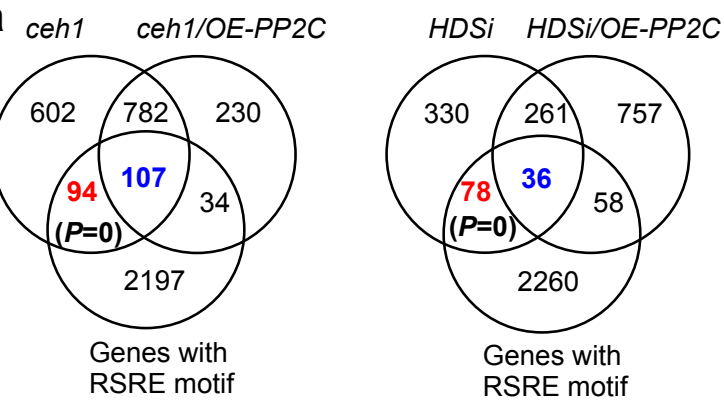

b

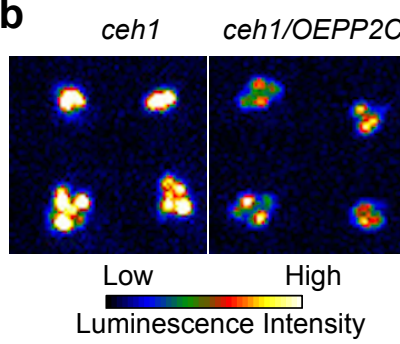

C
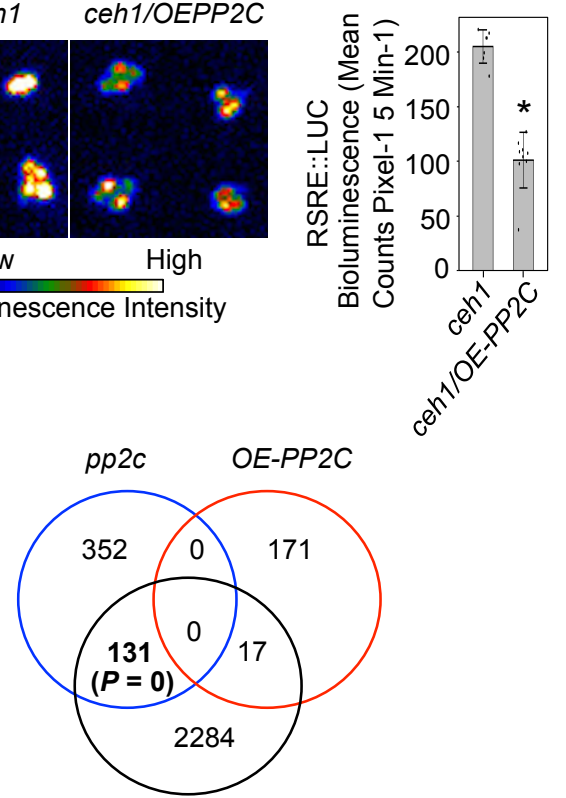

Genes with RSRE motif

Figure 3. PP2C suppresses the MEcPP-inducible SAR and RSRE-containing genes

(a) Venn diagrams illustrate suppression of a significant number of MEcPP-inducible RSRE-motif containing genes in PP2C overexpressing (ceh1/OE-PP2C and HDSi/OE-PP2C) lines. (b) Representative images of $P P 2 C$ overexpressing lines in the ceh1 background (ceh1/OE-PP2C) show the notable reduction of RSRE: LUC activity compared to the ceh1 mutant. The bar underneath displays the intensity of LUC activity and the histogram show quantification of LUC activity. Data are presented as means \pm SEM. Asterisk notes statistically significant differences $(P \leq 0.05)$ by using two-tailed Student's $t$ test. (c) Venn diagrams illustrate enrichment of RSRE-motif containing genes whose expressions are significantly induced in the $p p 2 c$ mutant but not in OE-PP2C plants under standard conditions. 
a rip and NHP biosynthesıs<smiles>CC(CCCCCCCCCCCCCCCCC(=O)O)C(=O)O</smiles><smiles>[R6][R6]1CC=C(C(=O)O)NC1</smiles>

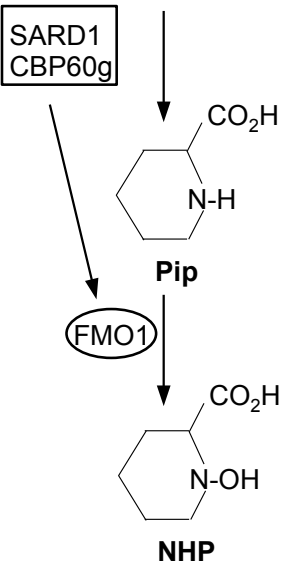

b
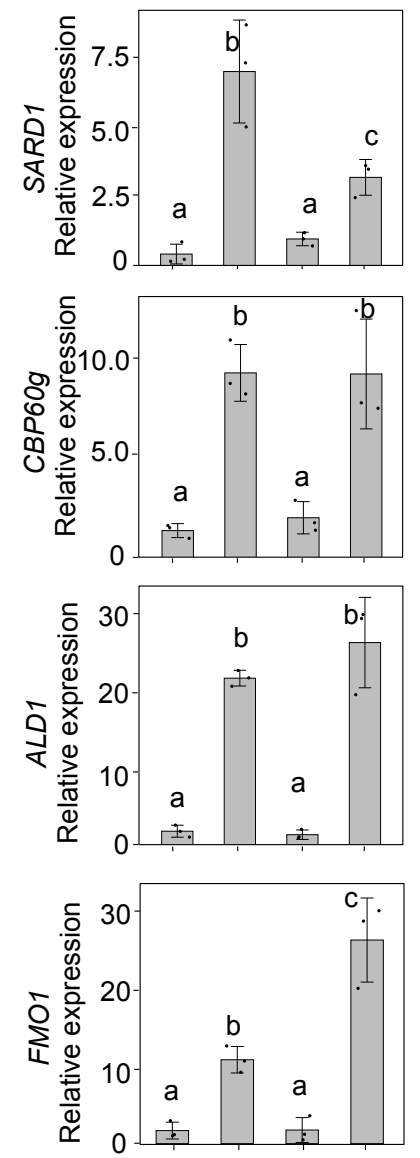

C
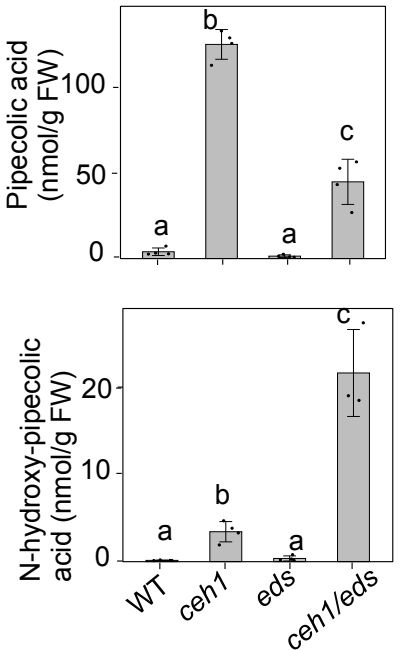
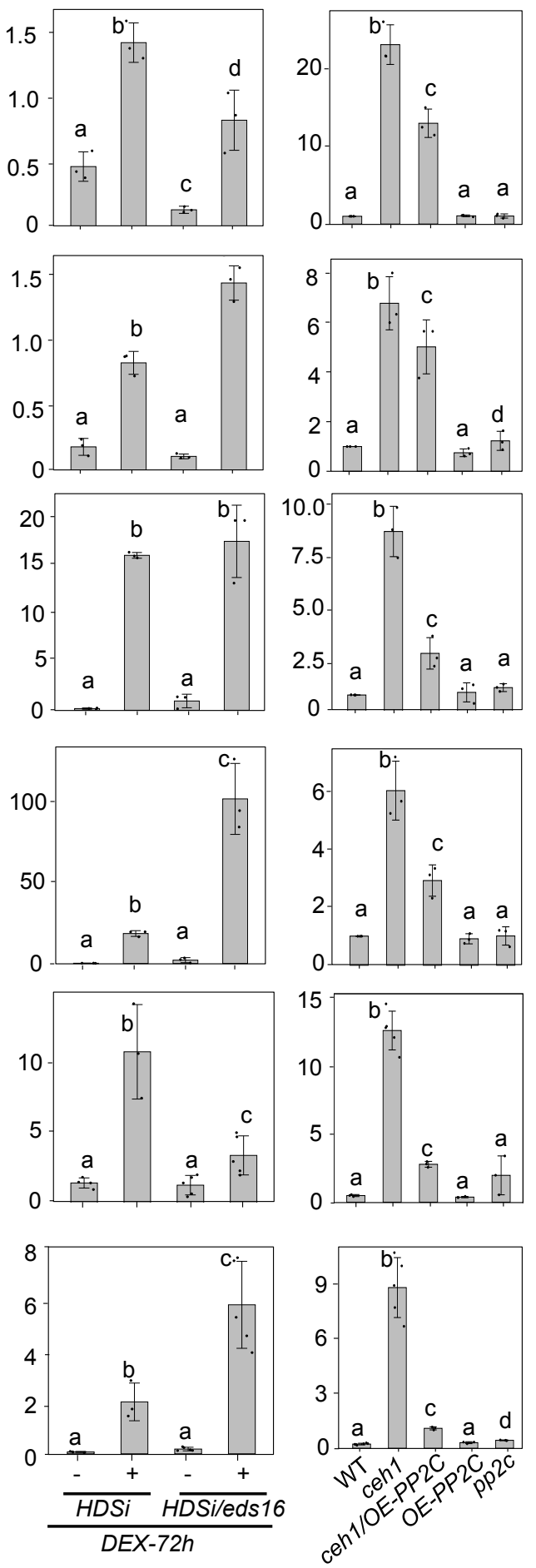

Figure 4. PP2C suppresses transcription of Pip and NHP biosynthesis genes and corresponding metabolites

(a) Schematic presentation of pipecolic acid (Pip) and N-hydroxy-pipecolic acid (NHP) biosynthesis pathway. (b) Relative expression levels of SARD1, CBP60g, ALD1 and FMO1 in WT, ceh1, eds16, ceh1/eds16, and in mock (-) and post $72 \mathrm{~h}$ DEXtreated HDSi and HDSi/eds16 lines, and in PP2C overexpression in the wild type (OE-PP2C) and the ceh1 mutant (ceh1/OE$P P 2 C$ ) backgrounds as well as in pp2c mutant, show reversion of SA- and MEcPP-dependent induction of these genes in PP2C overexpressing lines. (c) Measurements of Pip and NHP metabolites in aforementioned genotypes confirm their MEcPP- and SA-dependent alterations, and their reduced abundance in PP2C overexpression lines. All Data are mean \pm SD of three biological and three technical replicates. Lower case letters on top of histograms represent statistically significant differences $(P$ $\leq 0.05)$ by using ANOVA test. 

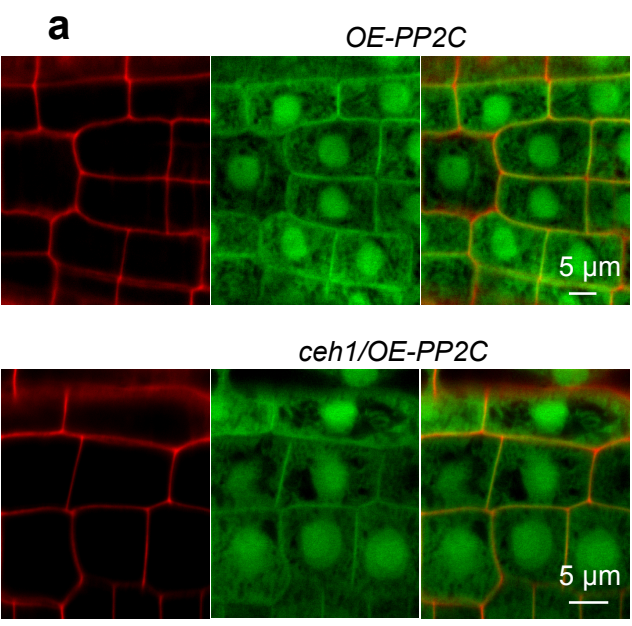

C

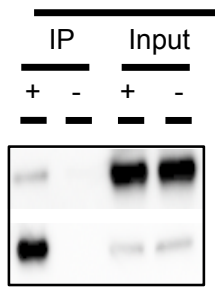

IP: GFP-Trap
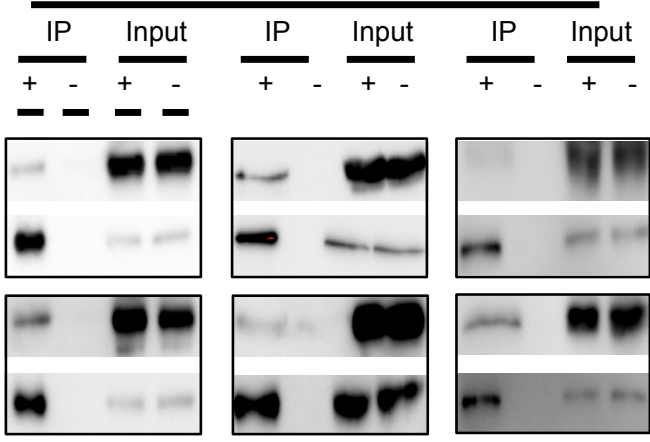

ceh1/OE-PP2C
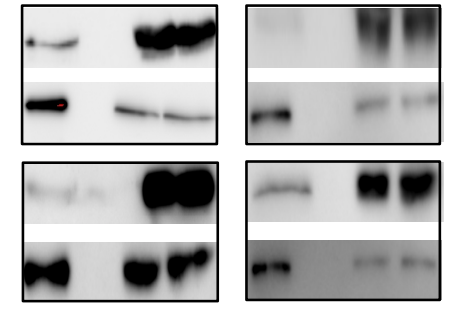

a-MAPK6

a-GFP

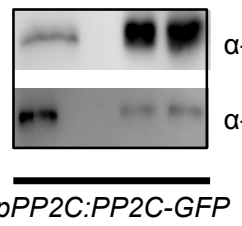

a-MAPK3

a-GFP b
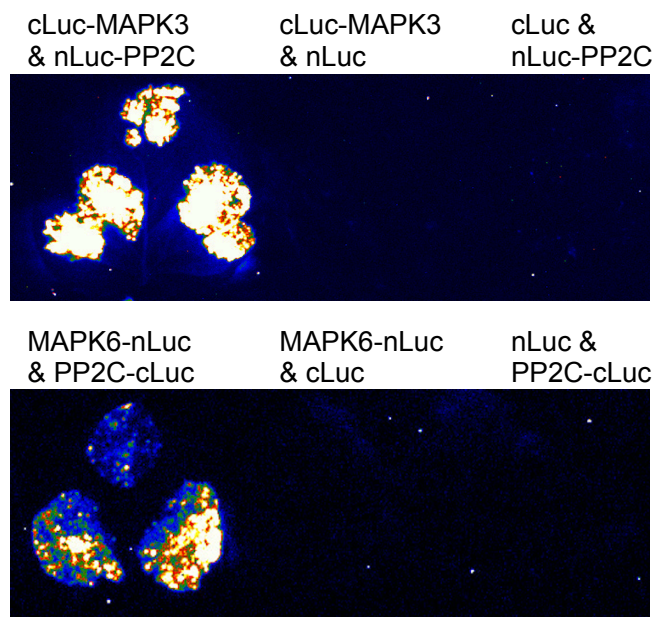

d
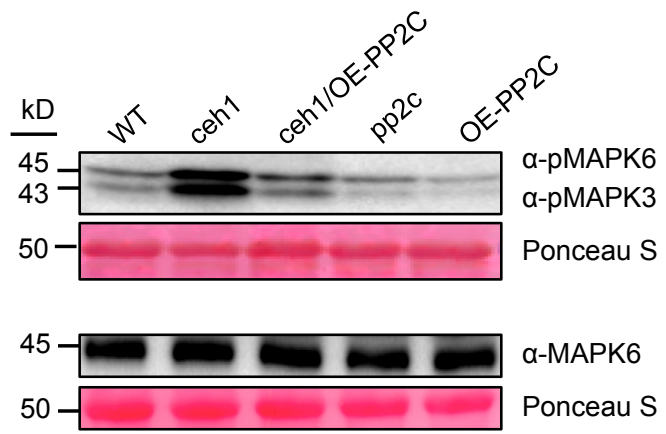

a-MAPK6

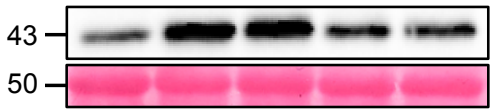

Ponceau S

a-MAPK3

Ponceau S

Figure 5. MAPK3/6 phosphorylation levels and their physical interaction with PP2C

(a) Confocal images of plasma membrane, cytosolic and nuclear localization of PP2C in the wild-type (OE-PP2C) and ceh1 (ceh1/OEPP2C) backgrounds overexpressing 35S::PP2C-GFP construct. (b) Representatives of split luciferase complementation assays in Nicotiana benthamiana displayed by dark-field images of leaves expressing cLuc-MAPK3 (C-terminal Luc fused with MAPK3) and nLuc-PP2C (N-terminal Luc fragment fused with PP2C) (upper panel) and MAPK6-nLuc (MAPK6 fused with N-terminal fragment of Luc) and PP2C-cLuc (PP2C fused with C-terminal fragment Luc) (lower panel). (c) The in vivo interaction of PP2C with MAPK3 and MAPK6 determined by co-immunoprecipitation assay. Protein samples obtained from ceh1/OE-PP2C, OE-PP2C, and $p P P 2 C: P P 2 C-$ GFP seedlings grown under standard conditions were immunoprecipitated using GFP (+) and empty (-) magnetic beads. Immunoblots were analyzed using with a-MAPK3 or a-MAPK6. Each blot shows protein inputs before (input, right panels) and after (IP, left panels) immunoprecipitation. (d) Immunoblots show that PP2C overexpression in the ceh1 mutant reverses the MEcPP-mediated high phosphorylation of MAPK3 and MAPK6 ( $\alpha$-pMAPK6 and $\alpha$-pMAPK3, top panels), without notable impact on the protein abundance of MAPK6 (middle panels) or MAPK3 (bottom panels). Ponceau S staining shows protein loading. 
a

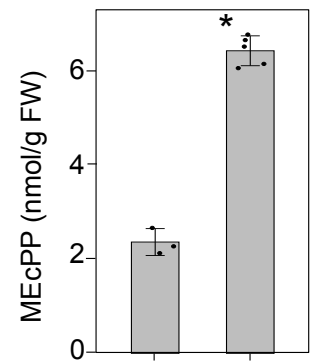

High light b

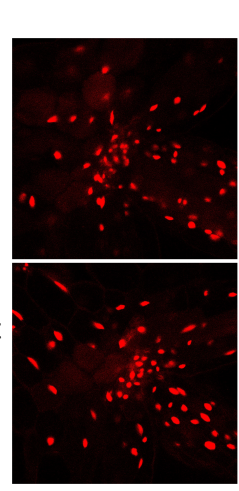

R2D2

High light
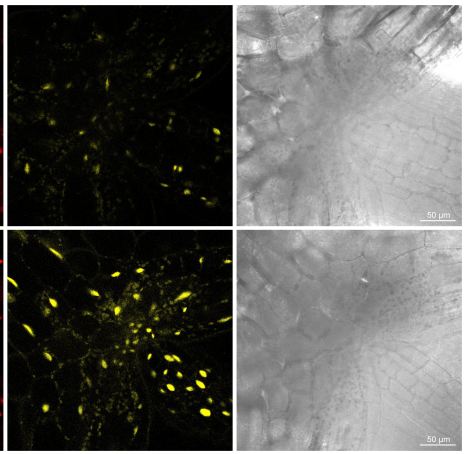

C

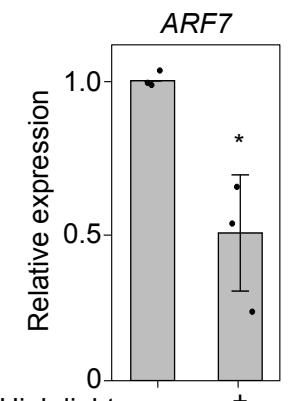

High light

WT
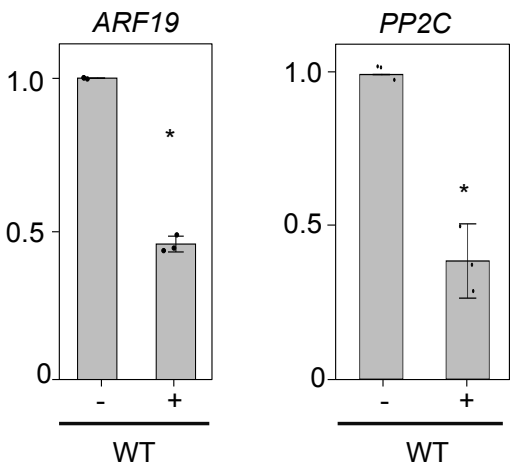

d

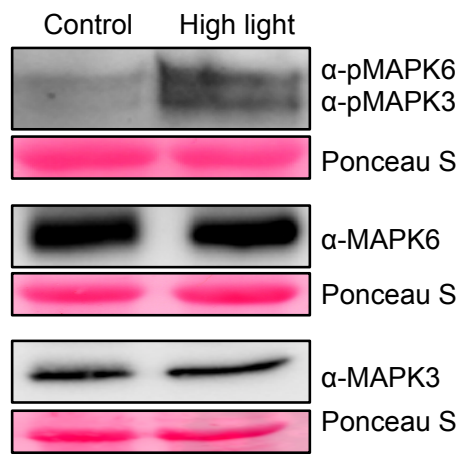

e

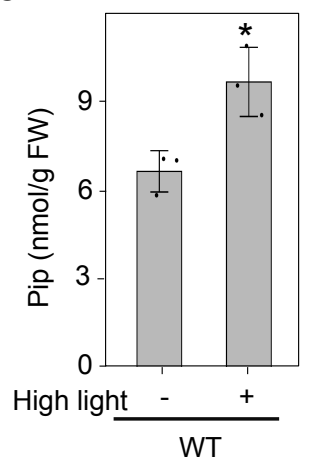

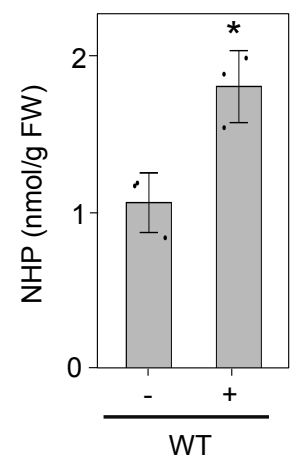

Figure 6. High light induces MEcPP accumulation and enhances Pip and NHP abundance

(a) Induction of MEcPP levels and (b) reduction of IAA abundance 90 min post high light ( $800 \mu \mathrm{mol} \mathrm{m}^{-2} \mathrm{~s}^{-1}, 90 \mathrm{~min}^{2}$ treatment as measured by fluorescence of mDII-ntdTomato/DII-n3xVenus and the corresponding bright-field images of control and high light-treated auxin reporter R2D2 seedlings. (c) Relative expression levels of ARF 7/ 19 and PP2C in high light-treated seedlings. (d) Immunoblot analyses of total and phosphorylated MAPK3/6 in high light-treated seedlings. Ponceau S show equal protein loading. (e) Enhanced levels of Pip and NHP metabolites in high light-treated seedlings. Two-tailed Student's $t$ tests and ANOVA tests are used for the statistical analyses and the asterisk and different letters denote significance $(P \leq 0.05)$. 
a

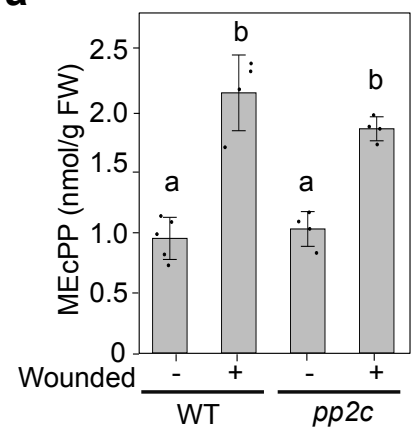

b

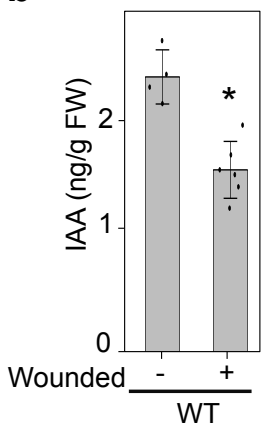

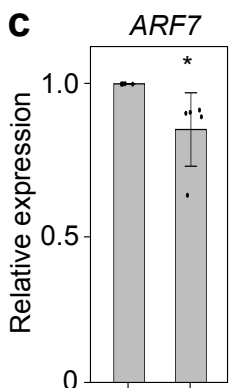

Wounded

ed

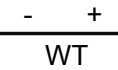

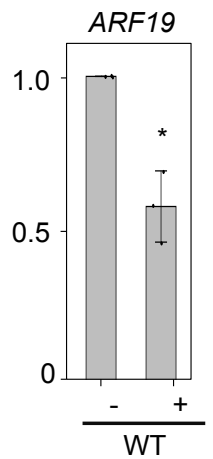

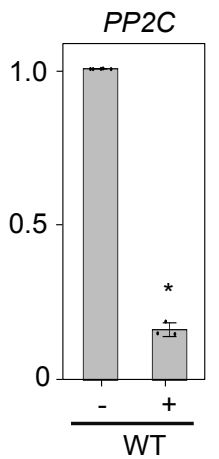

d
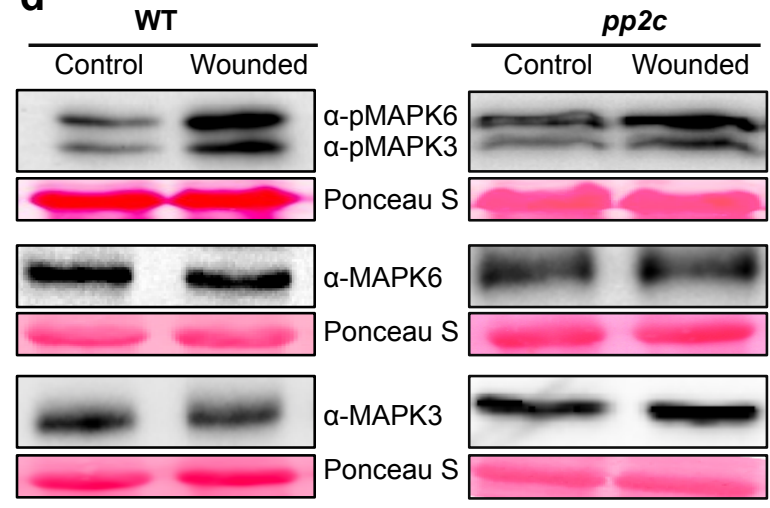

$\mathbf{e}$

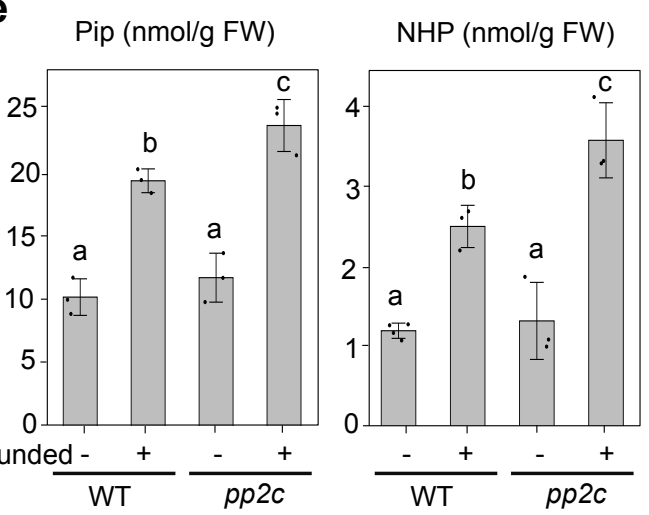

Figure 7. Wounding induces MEcPP accumulation and enhances Pip and NHP abundance

(a) Induction of MEcPP levels and (b) reduction of IAA abundance in 90 min post wounded WT and $p p 2 c$ mutant seedlings compared to control unwounded plants. (c) Relative expression levels of $A R F 7 / 19$ and $P P 2 C$ in unwounded and wounded WT plants. (d) Immunoblot analyses of total and phosphorylated MAPK3/6 in unwounded and wounded WT and pp2c mutant seedlings. Ponceau S show equal protein loading. (e) Metabolic analyses of Pip and NHP in wounded and WT and pp2c mutant seedlings. Two-tailed Student's $t$-tests and ANOVA tests are used for the statistical analyses and the asterisk and different letters denote statistical significance $(P \leq 0.05)$. 
a

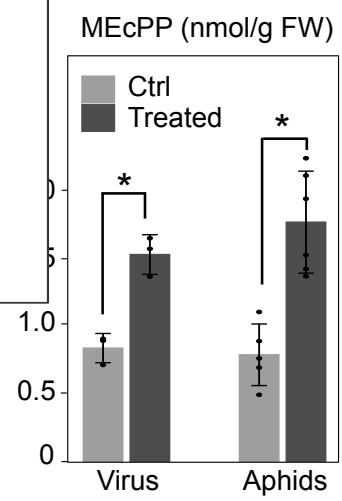

b

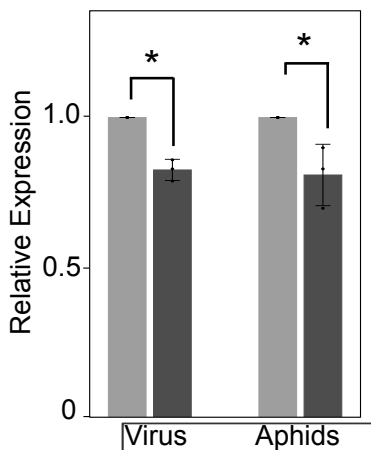

ARF19

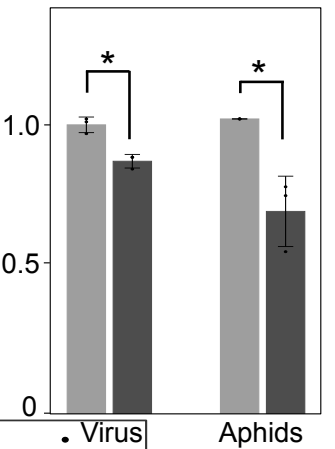

$P P 2 C$

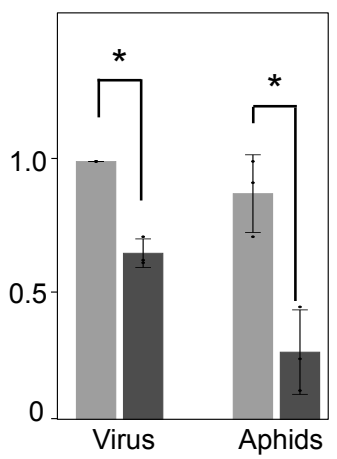

C

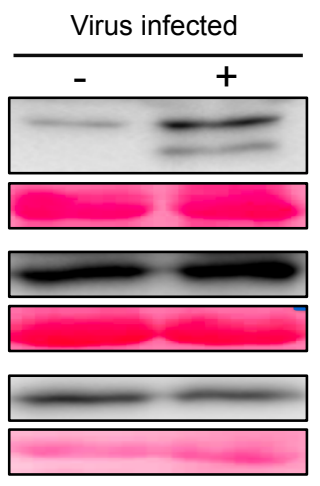

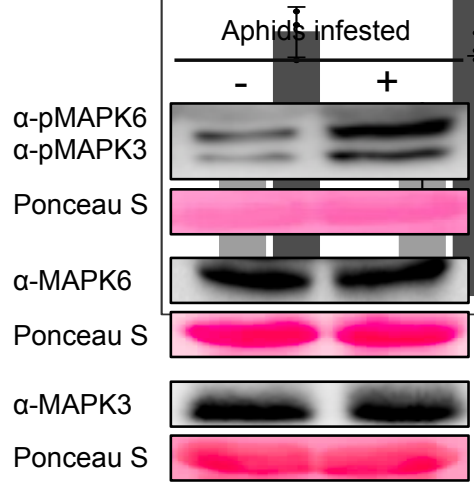

d

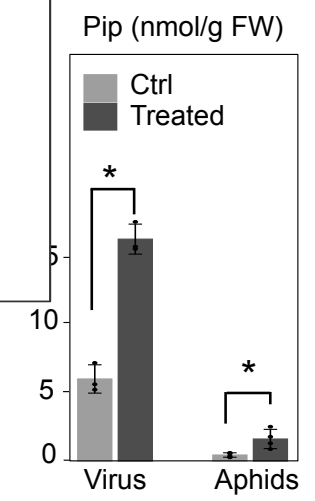

$\mathrm{NHP}(\mathrm{nmol} / \mathrm{g} \mathrm{FW})$

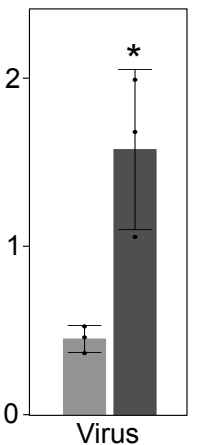

Figure 8. Aphid infestation and viral infection induce MEcPP accumulation and enhance Pip and NHP abundance (a) Induction of MEcPP post 2 weeks of cucumber mosaic virus (CMV-M2b) infection, and 24h aphid infestation in WT seedlings, and (b) suppression of $A R F 7 / 19$ and $P P 2 C$ expression in biotically challenged plants. (c) Immunoblot analyses of total and phosphorylated MAPK3/6 in mock (-) and virus/aphids (+) treated seedlings. Ponceau $S$ show equal protein loading. (d) Metabolic analyses of Pip and NHP in mock (-) and virus/aphids (+) treated seedlings. The asterisk denotes statistical significance $(P \leq 0.05)$ by using two-tailed Student's $t$-tests. 


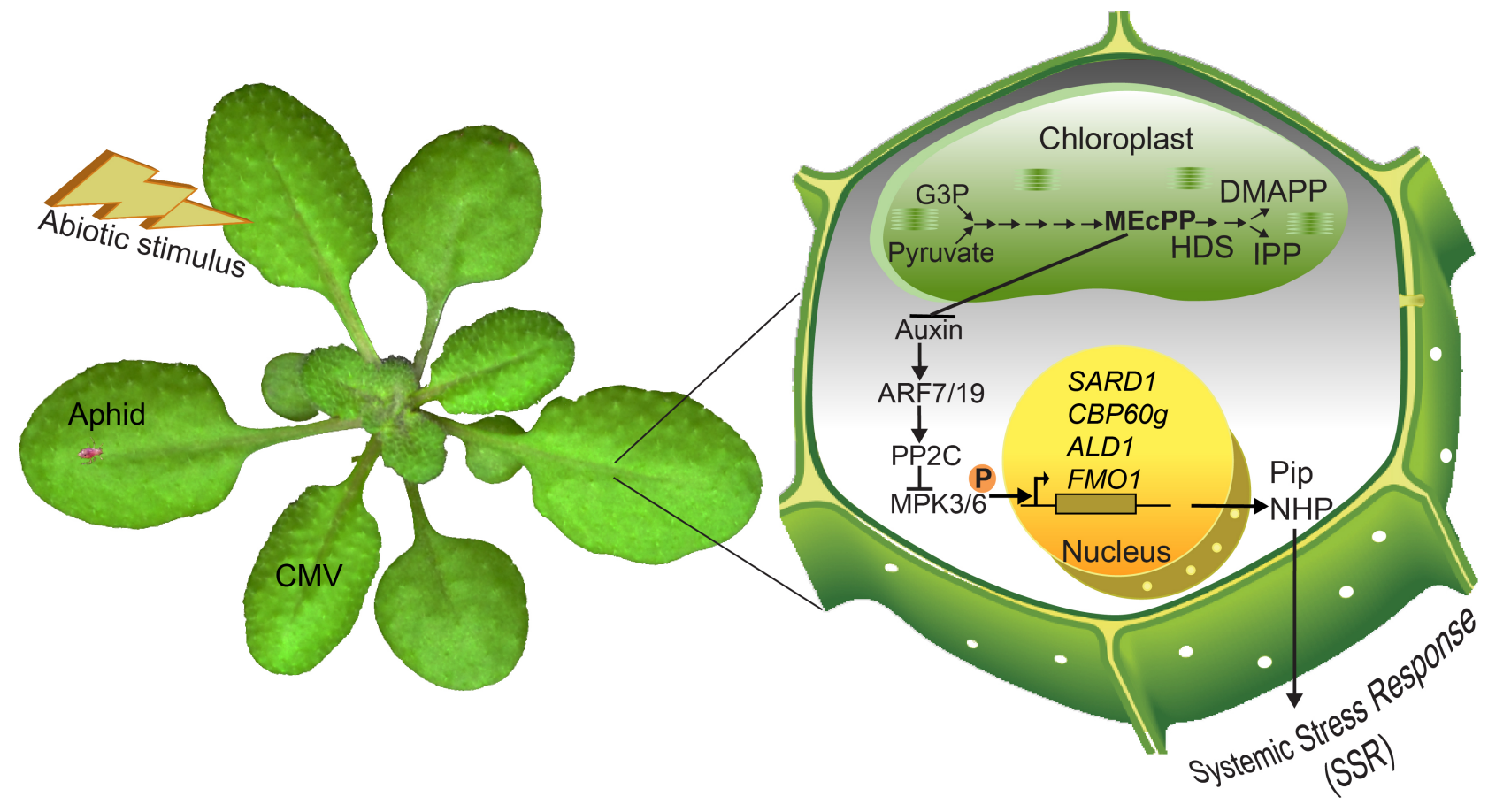

Figure 9. Biotic and abiotic insults trigger the retrograde signaling cascade initiating SSR

Schematic model depicting biotically and abiotically-induced MEcPP-accumulation mediates reduction of auxin abundance that lessens expression levels of the ARF7/19, the transcriptional activators of PP2C. This enables phosphorylation of MAPK3/6 required for induction of Pip and NPH biosynthesis genes and production of their respective metabolites key to activation of general SSR. 